\title{
Kolloid-Zeitschrift
}

Zeitschrift für wissenschaftliche und technische Kolloidchemie (iraher "Zeitschrift far Chemie und Industrie der Kolloide“)

Organ für das Gesamtgebiet der reinen und angewandten Kolloidchemie Herausgegeben von

Prof. Dr. Wolfgang Ostwald in Leipzig, Brandvorwerkstrabe 77

Erschetnt monatich i mal Verlag von THEODOR STEINKOPFF Drosdon und helpzigs

\section{Zur Kenntnis der Stabilitätsverhältnisse bei Platin-Organosolen.}

\author{
Von Johs. Lindeman (Kristiania) und The Svedberg (Upsala).
}

(Eingegangen am 16. Febr. 1921.)

Platin-Organosole sind bisher wenig studiert. The Svedbergl) hat Sole mit Diäthyläther ais Dispersionsmittel untersucht, indem der Aether kleine Mengen Wasser oder Aethylalkohol enthielt.

Er zeigte, dab diese Sole wenig stabil waren. Während der Darstellung (bei Zerstäubung mit oszillatorischen Entladungen) trat of Koagulation ein. Durch Zerstäuben bei niedriger Temperatur konnte diese jedoch vermieden werden; aber das Sol koagulieite dann oft, wenn die Temperatur erhöht wurde. Aus den experimentellen Daten ging hervor, dab, wenn das Sol koagulierle, dies bei einer Temperatur, welche für das betreffende Sol charakteristisch war, geschah, eine Temperatur, die er die kritische Temperatur nannte.

Er zeigte weiter, daB Wasser, Alkohol und Aether verschieden auf die Stabilität wirkten; ein Zusatz yon Wasser und Alkohol wirkte stabilisierend, während eine Erhöhung des Aethergehalts eine entgegengesetzte Wirkung hatte. Dies äuBerte sich dadurch, daß die kritische Temperatur mit zunehmender Konzentration von $\mathrm{H}_{2} \mathrm{O}$ resp. $\mathrm{C}_{2} \mathrm{H}_{5} \mathrm{OH}$ höher wurde, je der Gehalt dieser beiden Stoffe brauchte gar nicht sehr grob $\mathrm{zu}$ sein, damit die Koagulation selbst beim Siedepunkt des Dispersionsmittels überhaupt nicht eintrat.

Bei diesen Versuchen ist also die Koagulationstemperatur gemessen, um einen Einblick in den Stabilitätszustand der Sole zu bekommen. Die durchschnittliche Partikelgröße kann auch

3) The Svedberg, Koll.-Zeitschr. 2, 142 (1907/1908). hierbei angewendet werden, una $S$ ved berg und Inouye ${ }^{2}$ ) haben dieses später kurz getan; sie zähiten die Partikelanzahl im Ultramikroskop vor und während der Koagulation.

Die vorliegende Arbeit sehandelt die Stabilitätsverhältnisse bei Platinsolen mit AetherAlkoholmischungen als Dispersionsmittel. Teils sind langsame Koagulationen mit Hilre von Partikelgröbebestimmungen untersucht worden, teils schnelle Koagulationen durch Messungen der kritischen Temperatur. In beiden Fällen zeigen die Versuche, daß man annehmen kann, dab diese Sole im Laufe der Zeit sich auf der vollständigen Koagulation Đăhern, ohne dab irgendwelche Einwirkung von äuBeren Faktoren nachgewiesen werden kann. Dieser Prozeb, diese Wanderung gegen die vollständige Koagulation, wird in folgendem die spontane Koagulation genannt. Weiter ist es Gegenstand der Untersuchung, ob man die Schnelligkeit der spontanen Kongulation durch Aenderung der äuberen Paktoren beeinflussen kann.

Hierzu ist $z u$ bemerken, dab man fruher oft die Erfahrung gemacht hat, dab Sole, auf elektrischem Wege hergestellt, nicht haltbar sind, sondern nach einer gewissen Zeit koagulieren.

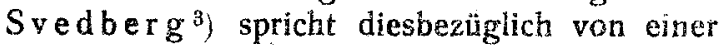
Selbstkoagulation.

1. Die Anwendung der Keimmethode auf Platinsole.

Bei den untersuchten Solen war die Partike? größe so klein, dâ eine direkte Zählung in

2) The Svedberg u. K. Inouye, Koll.Zeitsch. 9. 153 (1911).

g) The Svedberg, loc. cit. 
Uitramikroskop nicht möglich war. G. Börjeson ${ }^{4}$ hat indessen gezeigt, dab R. Zsigmondy's ${ }^{5}$ ) Keimverfahren (Doerinckel's ${ }^{6}$ ). Methode mit $\mathrm{H}_{2} \mathrm{O}_{2}$ ) dahin erweitert werden kann, daf nicht allein Goldsole, sondern auch andere Sole als Keimflüssigkeit benutzt werden können. Er hat u. a. qualitativ gezeigt, daß Gold sich auf Platinkeime niederschlägt, wenn $\mathrm{HAuCl}_{4}$ mit $\mathrm{H}_{2} \mathrm{O}_{2}$ reduziert wird. Für Kadmium und einige andere Metalle hat er weiter gezeigt, dab es eine Proportionalität gibt zwischen hinzugesetzten Volumen Keimflüssigkeit und gebildeten Au-Partikeln, gleich früheren Versuchen von A. Westgren ${ }^{7}$ ) mit Au-Solen als Keimflüssigkeit.

Die vergoldeten Platinpartikel können leicht so grob gewonnen werden, daß sie eine meßbare Lănge, z. B. von einem Tage zum andern sedimentieren, und S tokes' Formel gibt dann, Kugelform vorausgesetzt, den Radius $R$ der Partikel. Der Radius $r$ der primären Pt-Partikel kann nun berechnet werden, wenn man die Masse Gold g, die ausreduziert ist, und die Masse $p$ der vorhandenen Pt-Keime kennt; nämlich

$$
\mathbf{r}^{3}: \mathbf{R}^{3}=\mathrm{s}_{\mathrm{g}} \mathrm{P}: \mathrm{s}_{\mathrm{p}} \mathrm{g}
$$

wo $s_{p}$ und $s_{g}$ das spezifische Gewicht von beziehungsweise Platin und Gold sind. Das Verhältnis $\frac{s_{\mathrm{p}}}{s_{\mathrm{g}}}=\frac{21,3}{19,4}$ weicht indessen so wenig von $1 \mathrm{ab}$, daf dessen dritte Wurzel gleich 1 gesetzt werden kann, und wir haben somit

$$
r=\sqrt[3]{\frac{p}{g}} \cdot R
$$

Bei der vorliegenden Arbeit sind eine Anzahl Partikelgrößebestimmungen nach diesem Prinzip vorgenommen worden, teils von Solen mit absolutem $\mathrm{C}_{2} \mathrm{H}_{5} \mathrm{OH}$, teils mit Mischungen von $\mathrm{C}_{2} \mathrm{H}_{5} \mathrm{OH}$ und $\left(\mathrm{C}_{2} \mathrm{H}_{5}\right)_{2} \mathrm{O}$ als Dispersionismittel. Die Sole wurden alle nach $S$ vedberg's ${ }^{8}$ ) Methode mit oszillatorischen Entladungen dargestellt (näheres Abschnitt 4). Die Versuche sind folgendermaßen ausgeführt: Gewöhnlich ist $1 \mathrm{ccm}$ Pt-Sol herauspipettiert, entweder direkt aus dem ursprünglichen Sol oder aus einem Sol, welches durch Verdünnung mit

4) G. Börje s on, Koll, - Zeitschr. 27, 18 (1920). 5) R. Zs i g mon dy, Zeitschr, f. physik. Chem. 56, 65 (1906).

6) Fr. Doerincke1, Zeitschr. f. anorg. Chem. 63, 344 (1909).

7) A. Westgren, Zeitschr. f. anorg. Chem. 93, 151 (1915).

8) The S vedber g, Koll.-Zeitschr. 1, 229 (1906/07).
$\mathrm{C}_{2} \mathrm{H}_{5} \mathrm{OH}$ gewonnen ist. (Betreffs Bestimmung der Konzentration des Sols siehe Abschnitt 4.) Enthält das Platinsol Aether, so wird das herausgenommene Volum meist mit einigen Kubikzentimeter Alkokol verdünnt. Eine bestimmte Menge $(2,5-5 \mathrm{ccm}) \mathrm{H} \mathrm{Au} \mathrm{Cl}_{4}$-Lösung ca. $10^{-2}$ normal (genau bestimmt durch Reduktion von $25 \mathrm{ccm}$ mit $\mathrm{NH}_{2} \mathrm{OH} . \mathrm{HCl}$ und folgendem Wägen des ausreduzierten Goldes) wurde in ein andercs Gefäß gebracht, mit besonders reinem Wasser verdünnt und zu dem herausgenommenen Volum Pt.Sol gegossen. Die Wassermenge wurde so variiert, dab das Cesamtvolum stets $50 \mathrm{ccm}$ war. Schlieflich wurden drei Tropfen Merck's Perhydrol hinzugefügt und alles gut umgerührt. Nachdem die Reduktion beendet war, wurde das Sol in zylindrischen Gefäßen in einen Raum mit konstanter Temperatur $\left(14-15^{\circ}\right)$ zur Sedimentation gestellt. In einigen Fällen war die GröBe der Partikel der auf diese Weise hergestellten AuSole so klein, daß die sedimentierte Strecke von einem Tage zum andern zu kurz war, um eine genaue Abmessung zu gestatten. Solche Partikel wurden dann nochmals vergoldet. $5 \mathrm{ccm}$ eines solchen Sols (d. h. ein Zehntel des Volums) wurde neues $\mathrm{HAuCl}_{4}+$ Wasser (so dab das gesamte Volum $50 \mathrm{ccm}$ wurde) und neues $\mathrm{H}_{2} \mathrm{O}_{2}$ hinzugesetzt; nun wurde das Ganze dem Sedimentieren überlassen.

Nach ungefähr 20 Stunden wurde die sedimentierte Strecke der obersten Teilchenschicht abgemessen. Die Sedimentationsgeschwindigkeit $\mathrm{V}$ in Millimeter pro Stunde ist nun gefunden, und der Radius $R$ der vergoldeten Partikel ist nach Stokes' Formel

$\mathrm{zu}$ berechnen. Hier ist

$$
\mathrm{R}^{2}=\frac{9}{2} \frac{\mathrm{v} \cdot \eta}{\mathrm{g}\left(\mathrm{s}_{2}-\mathrm{s}_{2}\right)}
$$

g die Beschleunigung der Schwere $981 \mathrm{~cm}$ (Upsala),

$s_{1}$ das spezifische Gewicht der sedimentierten Partikel. Da der Platingehalt derselben nie 3 Proz. übersteigt, wird $s_{1}$ dem spezifischen Gewicht des Goldes gleichgestellt, d. i. 19,4 (Averkieff),

$s_{2}$ das spezifische Gewicht der Flüssigkeit. Sie ist Wasser mit etwas Alkohol, aber nie über 8,4 Proz., dem entspricht ein spezifisches Gewicht 0,989. $s_{2}$ kann als konstant angesehen werden, gleich 1,0 , indem ein Fehler von 1 Proz. in $R^{2}$ vernachlässigt werden kann. Gleichfalls kann man von den kleinen Mengen Aether, die vorhanden sein kötnnen, absehen, 
v die Sedimentationsgeschwindigkeit in Zentimetern pro Sekunde, d. i.

$$
v=\frac{1}{10.3600} \mathrm{~V}
$$

$\eta$ die Viskosität der Flüssigkeit, ungefähr 0,01 . Ausgerechnet wird

$$
\begin{gathered}
\mathrm{R}=8,34,10^{-6} \sqrt{100 \eta} \cdot \sqrt{\mathrm{V}} \mathrm{cm} \\
=83,4 \sqrt{100 \eta} \cdot \sqrt{\mathrm{V}} \cdot \mu \mu .
\end{gathered}
$$

$\eta$ variiert sowohl mit der Temperatur als mit der Alkoholkonzentration. A. E. Dunstan und $\mathrm{F}$. B. Thol ${ }^{9}$ ) haben gezeigt, daB $\eta$ bei konstanter Temperatur mit der Alkoholkonzentration zwischen 0 und 20 Proz. linear wächst. Ihre Angaben betreffen $20^{\circ}, 25^{\circ}, 30^{\circ}$. Durch Extrapolation können die Werte des $\eta$ für die aktuelle Alkoholkonzentration bei den Sedimentationstemperaturen berechnet werden. Bei den vorliegenden Versuchen zeigt die Berech-

9) A. E. Dunstan u. F. B.Thole, Journ. Chem. Soc. $95,1556(1909)$. nung, daß die Werte des Paktors $100 \eta z$ wischen 1,09 (für 0,2 Proz. $\mathrm{C}_{2} \mathrm{H}_{5} \mathrm{OH}$ bei $14{ }^{\circ}$ ) und 1,30 (für 8,4 Proz. $\mathrm{C}_{2} \mathrm{H}_{5} \mathrm{OH}$ bei $15^{\circ}$ ) liegen. Die ganze Variation ist also 0,21 oder etwas unter 20 Proz., d. i. so grob, dab sie nicht vernachlässigt werden kann. Leider erlaubte die Zeit nicht, $\eta$ direkt $z u$ bestimmen, und die angewendeten berechneten Werte sind natürlich mit Fehlern behaftet. $\mathrm{Da}$ indessen die Versuchsfehler voraussichtlich eine Unsicherheit in dem Radius $r$ der Platinpartikel auf \pm 5 Proz. bewirken, mub man annehmen, dab die speziellen Werte eines jeden einzelnen Falles des verhălthismäBig wenig varilerenden Faktors $\sqrt{100 \eta}$ genügend genau für eine orientierende Uebersicht sind.

Zuerst sind einige Versuche vorgenommen worden, um vorzuführen, dat Platinalkosole (ohne Aether) als Keimflüssigkeit zur Ermittlung der Teilchengröße verwendet werden können. Die Resultate dieser Versuche sind in Tabelle I gegeben.

\section{Tabelle 1 .}

Sol A. In 100 Proz. $\mathrm{C}_{2} \mathrm{H}_{5} \mathrm{OH}$ zerstäubt. Pt-Konz. $15,5 \mathrm{mg}$ pro $100 \mathrm{ccm}$. Sedimentationsternperatur $14^{\circ}$.

$$
\text { Serie } 1 .
$$

3 Tage nach der Zerstäubung wurde ein verdünntes Sol aus 1 Teil Sol A und a) 4 Telle $\mathrm{C}_{\mathrm{g}} \mathrm{H}_{6} \mathrm{OH}$ abs. b) 9 Teile $\mathrm{C}_{\mathrm{g}} \mathrm{H}_{5} \mathrm{OH}$ abs. hergestellt. 6 Tage nach der Zerstăubung wurde $1 \mathrm{ccm}$ aus dem verdünnten Sole, d. i. a) $\left.p=31 \cdot 10^{-3} \mathrm{mg} \mathrm{Pt}, \mathrm{b}\right) \mathrm{p}=15,5 \cdot 10^{-3} \mathrm{mg}$ Pt herauspipettiert, und $\mathrm{g} \mathrm{mg} \mathrm{Au} \mathrm{herausteduziert.} \mathrm{(Vol.}$ $50 \mathrm{ccm}$.) Darnach Sedinentation eine Strecke $1 \mathrm{~mm}$ im Laufe von h Stunden.

$$
\text { Serie } 2 \text {. }
$$

13 Tage nach der Zerstäubung wurde $1 \mathrm{~cm}\left(\mathrm{c}=155 \cdot 10^{-3} \mathrm{mg} \mathrm{Pt}\right)$ von Sol A herauspipettiert, a ccm $\mathrm{C}_{8} \mathrm{H}_{6}$ OH hinzugesetzt, $\mathrm{g} \mathrm{mg} \mathrm{Au} \mathrm{in} \mathrm{einem} \mathrm{Volum} \mathrm{von} 50 \mathrm{ccm}$ herausreduziert. Hieraus wurde $5 \mathrm{ccm}$ genommen

\begin{tabular}{|c|c|c|c|c|c|c|c|c|c|}
\hline Serie & $\begin{array}{l}\text { a ccm } \\
\mathrm{C}_{2} \mathrm{H}_{6} \mathrm{OH} \\
\text { hinzu- } \\
\text { gesetzt }\end{array}$ & $\begin{array}{c}\mathrm{g} \mathrm{mg} \mathrm{Au} \\
\text { heraus- } \\
\text { reduziert }\end{array}$ & $\begin{array}{l}\mathrm{g}_{1} \mathrm{mg} \mathrm{Au} \\
\text { herans- } \\
\text { reduziert }\end{array}$ & $\begin{array}{c}\text { Prozent } \\
\mathrm{C}_{2} \mathrm{H}_{5} \mathrm{OH} \\
\text { bei der Sedi- } \\
\text { mentation }\end{array}$ & $\begin{array}{l}\sqrt{100 n} \\
\text { be- } \\
\text { rechnet }\end{array}$ & $\begin{array}{c}1 \mathrm{~mm} \\
\text { sedimen- } \\
\text { tierte } \\
\text { Strecke }\end{array}$ & $\begin{array}{l}\text { h Stunden } \\
\text { Sedimen-- } \\
\text { tations- } \\
\text { zeit }\end{array}$ & $\begin{array}{c}r \mu \mu \text { Teil- } \\
\text { chen } \\
\text { Radius } \\
\text { berechnet }\end{array}$ & $\begin{array}{l}\text { t Tage } \\
\text { Alter } \\
\text { des } \\
\text { Sols }\end{array}$ \\
\hline $\begin{array}{l}\text { la } \\
\text { lb }\end{array}$ & $\begin{array}{l}0 \\
0\end{array}$ & $\begin{array}{l}10,3 \\
10,3\end{array}$ & - & $\begin{array}{l}2,0 \\
2,0\end{array}$ & $\begin{array}{l}1,14 \\
1,14\end{array}$ & $\begin{array}{r}9 \\
14\end{array}$ & $\begin{array}{l}20,5 \\
20,5\end{array}$ & $\begin{array}{l}9,1 \\
9,0\end{array}$ & $\begin{array}{l}6 \\
6\end{array}$ \\
\hline $\begin{array}{l}2 \\
2 \\
2 \\
2 \\
2 \\
2\end{array}$ & $\begin{array}{l}0 \\
1 \\
4 \\
0 \\
2 \\
4\end{array}$ & $\begin{array}{l}9,6 \\
9,6 \\
9,6 \\
5,8 \\
5,8 \\
5,8\end{array}$ & $\begin{array}{l}9,6 \\
9,6 \\
9,6 \\
9,6 \\
9,6 \\
9,6\end{array}$ & $\begin{array}{l}0,2 \\
0,4 \\
1,0 \\
0,2 \\
0,6 \\
1,0\end{array}$ & $\begin{array}{l}1,09 \\
1,09 \\
1,11 \\
1,09 \\
1,10 \\
1,11\end{array}$ & $\begin{array}{l}10 \\
12 \\
10 \\
10 \\
10 \\
11\end{array}$ & $\begin{array}{l}20,5 \\
20,5 \\
20,5 \\
20,5 \\
20,5 \\
20,5\end{array}$ & $\begin{array}{l}7,2 \\
7,8 \\
7,4 \\
7,3 \\
7,4 \\
7,8\end{array}$ & $\begin{array}{l}13 \\
13 \\
13 \\
13 \\
13 \\
13\end{array}$ \\
\hline $\begin{array}{l}3 \\
3 \\
3\end{array}$ & $\begin{array}{l}0 \\
2 \\
4\end{array}$ & $\begin{array}{l}4,8 \\
4,8 \\
4,8\end{array}$ & $\begin{array}{l}9,6 \\
9,6 \\
9,6\end{array}$ & $\begin{array}{l}0,2 \\
0,6 \\
1,0\end{array}$ & $\begin{array}{l}1,09 \\
1,10 \\
1,11\end{array}$ & $\begin{array}{l}14 \\
15 \\
14\end{array}$ & $\begin{array}{l}19 \\
19 \\
19\end{array}$ & $\begin{array}{l}9,0 \\
9,4 \\
9,2\end{array}$ & $\begin{array}{l}14 \\
14 \\
14\end{array}$ \\
\hline
\end{tabular}
(d. i. ein Zehntel mit $15,5.10^{-3} \mathrm{mg}$ Pt, $\frac{\mathrm{g}}{10} \mathrm{mg} \cdot \mathrm{Au}$ und $\frac{\mathrm{a}+1}{10} \mathrm{C}_{2} \mathrm{H}_{5} \mathrm{OH}$ ), und $\mathrm{g}_{1} \mathrm{mg}$ Au herausreduziert. Darnach Sedimentation. $15,5 \cdot 10^{-3} \mathrm{mg} P t$ ist auf diese Weise mit $\left(\frac{\mathrm{g}}{10}+\mathrm{g}_{1}\right)$ ing Au vergoldet worden.

Serie 3 .

14 Tage nach der Zerstäubung wurde $1 \mathrm{ccm}$ herauspipettiert, und weiter wie in Serie 2 fortgefahren. 
Man sieht, dab diejenigen Versuche, die zur gleichen Zeit vorgenommen worden sind, übereinstimmende Werte für $r$ geben; dies mub dahin gedeutet werden, dab die hinzugefügten Platinpartikel als Keime fungiert haben. Ebenso ist ersichtlich, daf man, ehe man $\mathrm{HAuCl}_{4}$-Lösung zusetzt, bis zu $4 \mathrm{ccm}$ $\mathrm{C}_{2} \mathrm{H}_{5} \mathrm{OH}$ hinzugeben kann, ohne die Reduktion zu hindern. Aus Serie 2 der Tabelle bekommt man gleichfalls einen Eindruck über die Genauigkeit der Methode, indem dic extremen Werte +4 Proz. von dem Durchschnittswert 7,5 abs abweichen.

Man kan schlieben, daB Partikelgröben in platinsolen mit $\mathrm{C}_{2} \mathrm{H}_{5} \mathrm{OH}$ (absolut) a!s Dispersionsmittel nach der angewendeten Methode bestimmt werden können.

Enthalt das Sol Aether, ist es stets eine Schwierigkeit, Wasser hinzuzusetzen, chne dab Koagulation eintritt. Verdünnt man das Sol zuerst mit etwas Alkohol, kann eine sichtbare Koagulation beim Mischen mit Wasser ver. mieden werden. Es ist nicht leicht zu bestimnen, wie viele Male mehr Alkohol als Aether vorhanden sein mub, um Koagulation mit Wasser zu verhindern. Einige Vorversuche zeigten u. a., dab die Mischungsweise von Aether-Alkohol und Wasser von groBer Bedeutung ist, - die Koagulation tritt $z$. B. leichter ein, wenn man Aether-Alkohol zu Wasser giebt als umgekehrt. Daher wurde bei allen Versuchen Wasser zu Aether-Alkohollösung gegeben. Voraussichtlich kann irgendwelche Veränderung eines ätherhaltigen Sols, welches zum wässerigen Milieu überführt wird, nicht ganz vermieden werden. Die Aufgabe ist deshalb, diejenige Menge Alkohol zu finden, die hinreichend ist, um die Veränderung in $r$ praktisch urbedentend $z u$ machen. $Z u$ diesem Zwecke sind eine Reihe Versuche mit einem ätherfreien Sol, atessen $r$ bekannt ist, vorgenommen worden. Dem Sol wurden variierende Mengen Alkohol und $1 \mathrm{ccm}$ Aether (vor dem $\mathrm{HAuCl}_{4}$-Zusatz und der Reduktion) hinzugesetzt. Die Tabeile II zeigt die Ergebnisse.

Tabelle II.

Sol A. In 100 Proz. $\mathrm{C}_{2} \mathrm{H}_{5} \mathrm{OH}$ zerstäubt. Pt-Konz. $15,5 \mathrm{mg}$ pro $100 \mathrm{ccm}$. Sedimentationstemperatur $14^{\circ}$.

14 Tage (siche Tab. I Serie 3) nach der Zerstäubung wutde $1 \mathrm{ccm}(\mathrm{p}=155.10-3 \mathrm{mg}$ Pt) von Sol A herauspipetilert, a $\mathrm{cm} \mathrm{C}_{2} \mathrm{H}_{5} \mathrm{OH}$ hinzugesetzt, dann $1 \mathrm{ccm}$ Aether, $\mathrm{g} \mathrm{mg} \mathrm{Au}$ in einem Voin von $50 \mathrm{ccm}$ herausreduziert. Hieraus $5 \mathrm{ccm}$ genommen, $\mathrm{g}_{1} \mathrm{mg}$ Au herausreduziert. Darnach Sedimentation.

\begin{tabular}{|c|c|c|c|c|c|c|c|c|}
\hline $\begin{array}{c}\text { a ccm } \\
\mathrm{C}_{2} \mathrm{H}_{5} \mathrm{OH} \\
\text { hinzu- } \\
\text { gesetzt }\end{array}$ & $\begin{array}{l}\mathrm{g} \text { mg Au } \\
\text { heraus- } \\
\text { reduziert }\end{array}$ & $\begin{array}{c}g_{1} \mathrm{mg} \text { Au } \\
\text { heraus- } \\
\text { reduziert }\end{array}$ & $\begin{array}{c}\text { Prozent } \\
\mathrm{C}_{2} \mathrm{H}_{5} \mathrm{OH} \\
\text { bei der Sedi- } \\
\text { mentation }\end{array}$ & $\begin{array}{c}\sqrt{100} \eta \\
\text { be- } \\
\text { rechnet }\end{array}$ & $\begin{array}{l}1 \text { min } \\
\text { sedimen- } \\
\text { tierte } \\
\text { Strecke }\end{array}$ & $\begin{array}{c}\text { h Stunden } \\
\text { Sedimen- } \\
\text { tations- } \\
\text { zeit }\end{array}$ & $\begin{array}{c}\tau \mu \mu \text { Teil- } \\
\text { chen } \\
\text { Radius } \\
\text { berechnet }\end{array}$ & $\begin{array}{l}\text { Tage } \\
\text { Alter } \\
\text { des } \\
\text { Sols }\end{array}$ \\
\hline $\begin{array}{l}1 \\
2 \\
4 \\
4 \\
4\end{array}$ & $\begin{array}{l}4,8 \\
4,8 \\
4,8 \\
4,8 \\
4,8\end{array}$ & $\begin{array}{l}9,6 \\
9,6 \\
9,6 \\
9,6 \\
9,6\end{array}$ & $\begin{array}{l}0,4 \\
0,6 \\
1,0 \\
1,0 \\
1,0\end{array}$ & $\begin{array}{l}1,09 \\
1,10 \\
1,11 \\
1,11 \\
1,11\end{array}$ & $\begin{array}{l}14 \\
16 \\
15 \\
14 \\
16\end{array}$ & $\begin{array}{l}19 \\
19 \\
19 \\
19 \\
19\end{array}$ & $\begin{array}{l}9,0 \\
9,7 \\
9,4 \\
9,1 \\
9,8\end{array}$ & $\begin{array}{l}14 \\
14 \\
14 \\
14 \\
14\end{array}$ \\
\hline
\end{tabular}

Im Durchschnitt wird $\mathrm{r}=9,4 \mu \mu$ rit gröbter Abweichung der einzelnen Bestimmungen to $0,4 \mu \mu$ oder +4 Proz. In Tabelle I Serie 3 ist $r$ am seiben Tage für dasselbe Sol, aber ohne Zusatz von Aether bestimmt worden. Hier ist der Durchschnittswert $9,2 \mu \mu$. Der Aether bewirkt also eine Verschiebung desselben auf $0,2 \mu \mu$ oder 2 Proz., die gestattet werden kann.

Das Vorhandensein von Aether wirkt also nicht hindernd auf die Reduktion, und die Partikelgröben könrenbestimmt werden, selbst wenn A ether zugegen ist.

Voraussichtlich genügen zwei Volum Alkohol (cas eine Kubikzentimeter des Alkohols $+1 \mathrm{ccm}$ zugesetzter Alkohol) pro Volum Aether, um eine

nennenswerte Koagulation zu hindern. Bei den folgenden Versuchen ist jedoch gewöhnlich eine etwas gröhere Menge Alkohol verwendet worden, nie unter dem vierfachen Volum des Aethers, um ganz sicher zu sein. Es ist ja wahrschein. lich, dab Sole mit gröberen Partikeln als das oben erwähnte sich beirn Ueberführen in wässeriges Milieu mehr sensibel als dieses verhalten.

Man beachte, dab die Sedimentation bei den obigen Versuchen in völlig geschlossenet Gefäßen mit eingeschliffenen Ğlaspfropfen vorgenommen ist. Dies erwies sich als durchaus notwendig. Schon kleine Mengen Aether bewirken, dab die Sedimentation nicht stattinden kann, wenn offene Gefäßle, nur mit einer Glas platte bedeckt, gebraucht werden. Dieses ist 
Tabelle III.

\begin{tabular}{|c|c|c|c|}
\hline \multicolumn{2}{|c|}{$\begin{array}{c}\text { For der Kedulation } \\
\text { hinzugesetat }\end{array}$} & \multicolumn{2}{|c|}{ Sedimentation in } \\
\hline $\begin{array}{c}\text { cem } \\
\text { Aether }\end{array}$ & $\mid \mathrm{C}_{2} \mathrm{~B}_{5} \mathrm{OH}$ & $\begin{array}{l}\text { a) nur zugedeck ten } \\
\text { GefäBen }\end{array}$ & $\begin{array}{c}\text { b) ganz geschlossenen } \\
\text { Gefäßen }\end{array}$ \\
\hline 1,0 & 3,0 & $\begin{array}{l}\text { Sedimentierte nicht } \\
\text { in } 20 \text { Stunden. }\end{array}$ & - \\
\hline 0,5 & 1,5 & $\begin{array}{l}\text { Sedimentierte nicint } \\
\text { in } 20 \text { Stunden. }\end{array}$ & $\begin{array}{l}\text { Sediment. } 15 \mathrm{~mm} \\
\text { in } 19 \text { Stunden. }\end{array}$ \\
\hline 0,25 & 0,75 & $\begin{array}{l}\text { Sedimentierte nicht } \\
\text { in } 20 \text { Stunden. }\end{array}$ & $\cdots$ \\
\hline 0 & 0 & $\begin{array}{l}\text { Sediment: } 17,5 \mathrm{~mm} \\
\text { in } 20 \text { Sturden. }\end{array}$ & $\begin{array}{l}\text { Sediment. } 15 \mathrm{~mm} \\
\text { in } 19 \text { Stunden. }\end{array}$ \\
\hline
\end{tabular}

a) und b) können nicht miteinander verglichen werden. Die Versuche sind an verschiedenen Tagen vorgenommen.

auf die Verdampiung des Aethers und dadurch hervorgerufenen Strömungen in der Flüssigkeit zurückzuführen, und hat mit dem Reduktionsprozef selbst nichts $z u$ tun. Letzteres kann dadurch gezeigt werden, wenn zu einem Sol, welches schon reduziert und aus einem ätherfreien Platinsol gewonnen ist, Aether zugesetzt wird. Es wurde $z$. B. $0,5 \mathrm{ccm}$ Aether (in $1,5 \mathrm{ccri}$
Alkohol aufgelös) hinzugesetzt, und das Sol sedimentierte nicht, während ein gerau ebenso hergestelltes Sol ohne Aetherzusatz normal sedimentierte. Daß selbst kleine Mengen Aether eine Rolle splelen, zeigen folgende Versuche (in Tabelle III zusammengestellf), wo jedoch der Aether vor dem Zusatz der $\mathrm{H} \mathrm{Au} \mathrm{Cl}_{4}$ - Losung hinzugegeben wurdt.

Also scheint schon ein Gehaltron 0,5 Proz. Aether eine reguläre Sedimentation ohne besondere Vorsichts. mabregeln mit geschlossenen Gefähen unmöglich $z u$ nuachen.

2. Bestimmung you Partikelgroben in demselben Sol zu verschiedenen Zeiten ${ }^{10}$.

Die Untersuckung ist vorgenommen worden mit zwei Solen, Sol A und Sol B, in absolutem

10) Bestimmungen von Partikelgrößen bei anderen Metallorganosolen sind von $\mathbf{G}$. B örjeson gleich. zeitig vorgenommen. worden, vgl. Borjeson, Researches on the Electric Synthesis of Colloids, Diss. (Upsala 1921).

\section{Tabelle IV.}

Sol A. In 100 Proz. $\mathrm{C}_{2} \mathrm{H}_{5}$ OH zerstäubt. Pt-Konz. $15,5 \mathrm{mg}$ pro $100 \mathrm{ccm}$. Sedimentationstemperatur 140.

Serie 1.

t Tage nach der Zerstäubung wurde $1 \mathrm{~cm}(\mathrm{p}=155,10-3 \mathrm{mg} \mathrm{Pt})$ von Sol A herausgenommen, und im tibrigen wie in Tab. I Serie 2 vorgegangen. Für $t=13$ und $t=14$ ist der Durchschnitswert der dort angegebenen Versuche aufgezeichnet.

\section{Serie 2.}

3 Tage nach der Zerstäubung wurde ein verdünntes Sol aus 1 Teil Sol $A$ und a) 4 Teile $\mathrm{C}_{2} \mathrm{H}_{5} \mathrm{OH}$, b) 9 i'eile $\mathrm{C}_{2} \mathrm{H}_{5} \mathrm{OH}$ hergestellt. $t$ Tage nach der Zerstäubung wurde $1 \mathrm{ccm}$ ats dem verdïnnten Sole, $d$. $i$. a) $\left.\mathrm{p}=31.10^{-3} \mathrm{mg} \mathrm{Pt}, \mathrm{b}\right) 15,5.10^{-3} \mathrm{mg}$ Pt herauspipettiert und $\mathrm{g} \mathrm{mg} \mathrm{Au} \mathrm{herausreduziert.} \mathrm{Danach} \mathrm{Sedimentation.}$

Sol B. In 100 Proz $\mathrm{C}_{2} \mathrm{H}_{5} \mathrm{OH}$ zerstäubt. Pt-Konz. $33,5 \mathrm{mg}$ pro $100 \mathrm{ccm}$. Sedimentationstemperatur $14^{\circ}$.

Serie 1.

2 Tage nach der Zerstäubung wurde $1 \mathrm{ccm}$ von Sol $B$ heraisgenommen $\left(p=335,10^{-3} \mathrm{mg} P t\right)$, $\mathrm{g} \mathrm{mg}$ Au in eiriem Volum von $50 \mathrm{ccm}$ heráusreduziert. Hieraus wurde $5 \mathrm{ccm}$ genommen, $\mathrm{g}_{1} \mathrm{mg}$ Au herausreduziert. Dann Sedimentation.

Serie 2.

t Tage nach der Zerstäubung wurde 1 Teil Sol B mit 9 Teilen $\mathrm{C}_{2} \mathrm{H}_{5} \mathrm{OH}$ verdünnt. Hieraus wurde $1 \mathrm{ccm}$ genommen ( $\left.\mathrm{p}=33,5,10^{-3} \mathrm{mg} \mathrm{Pt}\right)$, $\mathrm{g} \mathrm{mg} A \mathrm{u}$ herausreduziert, danach Sedimentation.

Sol C. In einer Mischung von 50 Proz Aether und 50 Proz. $\mathrm{C}_{2} \mathrm{H}_{5} \mathrm{OH}$ zerstäubt.

Pt-Konz. 24,5 mg pro $100 \mathrm{ccm}$. Sedimentationstemperatur 140.

t Tage nach der Zerstäubung wurde 1 Teil Sol $\mathrm{C}$ mit 9 Teilen $\mathrm{C}_{2} \mathrm{H}_{5} \mathrm{OH}$ verdünt. Hiervon wurde $1 \mathrm{ccm}\left(\mathrm{p}=24,5.10^{-9} \mathrm{mg} \mathrm{Pt}\right)$ herausgenommen, $\mathrm{g} \mathrm{mg}$ Au herausreduziert, dann Sedimentation.

Sol D. In einer Mischung von 77 Proz. Aether und 23 Proz. $\mathrm{C}_{2} \mathrm{H}_{5} \mathrm{OH}$ zerstäubt. $\mathrm{Pt}$-Konz. $32,0 \mathrm{mg}$ pro $100 \mathrm{ccm}$. Sedimentationstemperatur $14^{\circ}$.

$$
\text { Serie } 1 .
$$

Kurze Zeit nach dem Schluß der Zerstăubung wurde $1 \mathrm{ccm}$ Sol D $\left(\mathrm{p}=320.10^{-3} \mathrm{mg}\right.$ Pt) heraus. genommen, $4 \mathrm{ccm} \mathrm{C}_{2} \mathrm{H}_{6} \mathrm{OH}$ hiazugesetzt, und $\mathrm{g} \mathrm{mg}$ Au in einem Volum von $50 \mathrm{ccm}$ herausreduziert. Hiervon wurde $5 \mathrm{ccm}$ genommen, $g_{1} \mathrm{mg}$ Au herausreduziert, danach Sedimentation.

$$
\text { Serie } 2 .
$$

$t$ Tage nach der Zerstäuoung wurde $1 \mathrm{ccm}$ Sol $D\left(p=320.10^{-3} \mathrm{mg}\right.$ Pt) herausgenommen: $4 \mathrm{ccm}$ $\mathrm{C}_{2} \mathrm{H}_{5} \mathrm{OH}$ hinzugesetzt. $\mathrm{g} \mathrm{mg} \mathrm{Au} \mathrm{herausreduziert,} \mathrm{danach} \mathrm{Sedimentation.}$ 
Tabelle IV.

\begin{tabular}{|c|c|c|c|c|c|c|c|c|c|}
\hline So! & $\begin{array}{l}g \mathrm{mg} \mathrm{Au} \\
\text { herăts- } \\
\text { reduziert }\end{array}$ & $\begin{array}{c}g_{1} \mathrm{mg} A u \\
\text { heraus- } \\
\text { reduziert }\end{array}$ & $\begin{array}{c}\text { Prozent } \\
\mathrm{C}_{3} \mathrm{H}_{5} \text { OH bei } \\
\text { der Sedi- } \\
\text { mentation }\end{array}$ & $\begin{array}{c}\sqrt{100 \eta} \\
\text { be- } \\
\text { rechnet }\end{array}$ & $\begin{array}{c}1 \mathrm{~mm} \\
\text { sedimen- } \\
\text { tierte } \\
\text { Strecke }\end{array}$ & $\begin{array}{c}\text { h Std. } \\
\text { Sedimen- } \\
\text { tations- } \\
\text { zeit }\end{array}$ & $\begin{array}{c}\mu \mu \text { Teil- } \\
\text { chen } \\
\text { Radius } \\
\text { berechnet }\end{array}$ & $\begin{array}{c}\text { Tage } \\
\text { Alter } \\
\text { des } \\
\text { Sols }\end{array}$ & Anmerkung \\
\hline $\begin{array}{l}\text { A } 1 \\
\text { A } 1 \\
\text { A } 1\end{array}$ & $\begin{array}{c}10,3 \\
=\end{array}$ & $\stackrel{10,3}{-}$ & $\stackrel{0,2}{-}$ & $\frac{1,09}{\square}$ & $\begin{array}{l}10 \\
- \\
-\end{array}$ & $\frac{20,5}{=}$ & $\begin{array}{l}7,1 \\
7,5 \\
9,2\end{array}$ & $\begin{array}{r}4 \\
13 \\
14\end{array}$ & $\begin{array}{l}\text { Siehe Tab.I S. } 2 \\
\text { Siehe Tab. IS. } 2\end{array}$ \\
\hline $\begin{array}{l}\text { A } 2 a \\
\text { A } 2 a \\
\text { A } 2 a\end{array}$ & $\begin{array}{l}10,3 \\
10,3 \\
10,3\end{array}$ & - & $\begin{array}{l}2,0 \\
2,0 \\
2,0\end{array}$ & $\begin{array}{l}1,14 \\
1,14 \\
1,14\end{array}$ & $\begin{array}{r}7 \\
9 \\
16\end{array}$ & $\begin{array}{l}20,5 \\
20,5 \\
21\end{array}$ & $\begin{array}{r}8,1 \\
9,1 \\
12,0\end{array}$ & $\begin{array}{r}4 \\
6 \\
10\end{array}$ & Siehe Tab. I S. 1 a \\
\hline $\begin{array}{ll}A & 2 b \\
A & 2 b \\
A & 2 b \\
A & 2 b\end{array}$ & $\begin{array}{l}10,3 \\
10,3 \\
10,3 \\
10,3\end{array}$ & $=$ & $\begin{array}{l}2,0 \\
2,0 \\
2,0 \\
2,0\end{array}$ & $\begin{array}{l}1,14 \\
1,14 \\
1,14 \\
1,14\end{array}$ & $\begin{array}{l}13 \\
14 \\
16 \\
11\end{array}$ & $\begin{array}{l}21 \\
20,5 \\
19 \\
21\end{array}$ & $\begin{array}{l}8,6 \\
9,0 \\
9,9 \\
7,8 ?\end{array}$ & $\begin{array}{r}5 \\
6 \\
7 \\
10\end{array}$ & Siehe Tab.1S.1b \\
\hline $\begin{array}{ll}\text { B } & 1 \\
\text { B } & 2 \\
\text { B } & 2 \\
\text { B } & 2 \\
\text { B } & 2\end{array}$ & $\begin{array}{l}7,9 \\
9,7 \\
9,7 \\
9,7 \\
9,7\end{array}$ & $\begin{array}{l}7,9 \\
= \\
=\end{array}$ & $\begin{array}{l}0,2 \\
2,0 \\
2,0 \\
2,0 \\
2,0\end{array}$ & $\begin{array}{l}1,09 \\
1,14 \\
1,14 \\
1,14 \\
1,14\end{array}$ & $\begin{array}{l}10 \\
10 \\
14 \\
14 \\
17\end{array}$ & $\begin{array}{l}22 \\
19 \\
19 \\
19,5 \\
20\end{array}$ & $\begin{array}{r}9,5 \\
10,4 \\
12,3 \\
12,2 \\
13,2\end{array}$ & $\begin{array}{r}2 \\
3 \\
5 \\
9 \\
15\end{array}$ & \\
\hline $\begin{array}{l}\mathrm{C} \\
\mathrm{C}\end{array}$ & $\begin{array}{l}9,7 \\
9,7\end{array}$ & - & $\begin{array}{l}1,9 \\
1,9\end{array}$ & $\begin{array}{l}1,14 \\
1,14\end{array}$ & $\begin{array}{l}14 \\
18\end{array}$ & $\begin{array}{l}21 \\
19\end{array}$ & $\begin{array}{l}10,7 \\
12,6\end{array}$ & $\begin{array}{l}1 \\
3\end{array}$ & \\
\hline $\begin{array}{l}\text { D } 1 \\
\text { D } 2 \\
\text { D } 2\end{array}$ & $\begin{array}{r}5,1 \\
10,2 \\
10,2\end{array}$ & $\begin{array}{l}10,2 \\
-\end{array}$ & $\begin{array}{l}0,8 \\
8,4 \\
8,4\end{array}$ & $\begin{array}{l}1,09 \\
1,30 \\
1,30\end{array}$ & $\begin{array}{r}26 \\
5 \\
21\end{array}$ & $\begin{array}{l}18,5 \\
21,5 \\
43\end{array}$ & $\begin{array}{l}15,6 \\
16,4 \\
23,5\end{array}$ & $\begin{array}{l}0 \\
2 \\
6\end{array}$ & \\
\hline
\end{tabular}

Sol D war nach ca. 14 Tagen vollstăndig koaguliert.

Alkohol zerstäubt und mit zwei Solen in einer Mischung von Aeiher und Alkohol zerstäubt, Sol C in 50 Proz. Aether und Sol D in 77 Proz. Aether. Die Resultate sind aus Tabelle IV ersichtlich.

Von einem Werte abgesehen, wächst $r$, die durchschnittliche Partikelgröße, mit der Zeit. Dies kann auf zweierlei Weise gedeutet werden. Man könnte annehmen, daß das angewandte Keimsol anfänglich Partikel von verschiedenen Dimensionen enthielt, wodurch die großen aber nicht die kleinen im Laufe der Zeit sedimentieren. Wird stets aus dem oberen Teil der Flüssigkeit herausgenommen, so nimmt die Anzahl der Platinkeime mit der Zeit stets $a b$; dies wird eine illusorische Vergröberung des berechneten Wertes von $r$ bewirken, wenn man immer mit der ursprünglichen Platinkonzentration rechnet (welches leicht ersichtlich ist?. Aber diese Annahme ist unwahrscheinlich, weil jedes Sol, ehe etwas herausgenommen, ein wenig durchgerührt wurde. Man wird besser annehmen können, dab Partikel in Uebereinstimmung mit v. Smoluchowski's ${ }^{11}$ ) Koagulationstheorie zu doppelten, dreifachen usw. zusammentreten. Die Anzahl der vergoldeten

11) M. v. Smoluch ow ski, Physik. Zeitschr. 17, 585 (1916); Zeitschr. f. physik. Chem. 92, 129 (1917),
Partikel pro Volumeinheit wird hiernach mit der Zeit $t a t s a c h l i c h$ geringer, welches einem größeren durchschnittlichen $r$ entspricht. Die Zunahme des $r$ mub deshalb als ein Ausdruck für faktische Veränderungen in dem Sole zwischen den verschiedenen Bestimmungen angesehen werden, also ein Ausdruck für e in e spontane Koagulation.

The Svedberg ${ }^{12}$ ) zeigte bei seinen Messungen der kritischen Temperatur, dab eine Vergrökerung der Aetherkonzentration in koagulierender Richtung wirkte. Diesbezüglich kann man erwarten, daf die Partikelgröbe schneller zunimmt, wenn man einem Sol Aether zusetzt; 'gleichfalls, dab die Teilchengröbe langsamer zunimmt, wernn: ein ätherhaltiges Sol mit Alkohol verdünnt wird. Daraufbin sind einige Versuche vorgenommen worden, siehe Tabelle $\mathrm{V}$.

Die Ergebnisse sind mit den Werten der ursprünglichen Sole verglichen worden. Man sieht, wie erwartet, das $r$ d a m schnellsten wächst, wo die Aetherkonzentration am gröbten ist.

In Fig. 1 sind die Werte der Tabellen IV und V eingezeichnet. Der Wert 7,8 $\mu \mu$ für Sol $A$ nach zehn Tagen weicht so viel von den übrigen $a b$,

12) The Svedberg, loc. cit. 
Tabelle V.

Sol B. In 100 Proz. $\mathrm{C}_{2} \mathrm{H}_{5} \mathrm{OH}$ zerstäubt. Pt-Konz, $35,5 \mathrm{mg}$ pro $100 \mathrm{ccm}$. Sedimentationstemperatur $14^{\circ}$.

2 Tage nach der Zerstäubung wurde 1 Teil Sol B 1 Teil Aether zugesetzt. $t$ Tage nach der Zerstäubung wurde $1 \mathrm{ccm}\left(\mathrm{p}=167,5 \cdot 10^{-3} \mathrm{mg} \mathrm{Pt}\right)$ dieses ätherischen Sols mit $4 \mathrm{ccm} \mathrm{C}_{2} \mathrm{H}_{5} \mathrm{OH}$ verdünnt, und hiervon wieder $1 \mathrm{ccm}\left(\mathrm{p}=33,5,10^{-3} \mathrm{mg} \mathrm{Pt}\right)$ herausgenommen, $\mathrm{g} \mathrm{mg} \mathrm{Au}$ herausreduziert, danach Sedimentation.

Sol C. In einer Mischung von 50 Proz. Aether und 50 Proz. $\mathrm{C}_{2} \mathrm{H}_{5} \mathrm{OH}$ zerstäubt.

Pt-Konz. $24,5 \mathrm{mg}$ pro $100 \mathrm{ccm}$. Sedimentationstemperatur $14^{\circ}$.

1 Tag nach der Zerstäubung wurde 1 Teil von Sol $\mathrm{C}$ mit 9 Teilen $\mathrm{C}_{2} \mathrm{H}_{5} \mathrm{OH}$ verdunnt. 3 Tage nach der Zerstäubung wurde aus dieser fünfprozentigen Aetherlösung $1 \mathrm{ccm}\left(p=24,5.10^{-3} \mathrm{mg} \mathrm{Pt}\right)$ genommen, $\mathrm{g} \mathrm{mg}$ Au herausreduziert, danach sedimentiert.

\begin{tabular}{|c|c|c|c|c|c|c|c|c|}
\hline Sol & $\mathrm{g} \mathrm{mg}$ & $\begin{array}{l}\text { Prozent } \\
\mathrm{C}_{2} \mathrm{H}_{5} \mathrm{OH}\end{array}$ & $\sqrt{100 \eta}$ & $1 \mathrm{~mm}$ & h St. & $r \mu_{\mu}$ & t Tage & Anmerkung \\
\hline $\begin{array}{l}\mathrm{B} \\
\mathrm{B}\end{array}$ & $\begin{array}{l}9,7 \\
9,7\end{array}$ & $\begin{array}{l}1,8 \\
1,8\end{array}$ & $\begin{array}{l}1,14 \\
1,14\end{array}$ & $\begin{array}{l}15 \\
22\end{array}$ & $\begin{array}{l}19 \\
19\end{array}$ & $\begin{array}{l}12,9 \\
15,4\end{array}$ & $\begin{array}{l}3 \\
5\end{array}$ & $\begin{array}{c}\text { Siehe Tabelle IV } \\
\# " \text { IV }\end{array}$ \\
\hline C & 9,7 & 1,9 & 1,14 & 15 & 19 & 11,6 & 3 & ", \\
\hline
\end{tabular}

Vergleichung mit gleichzeitigen Bestimmungen des $\mathrm{r}$ bei dem ursprünglichen Sol.

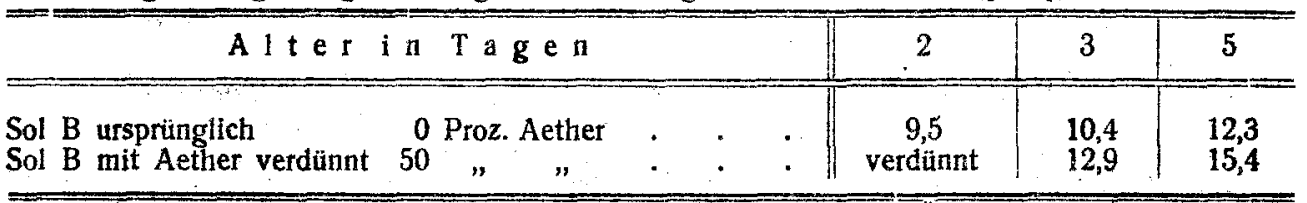

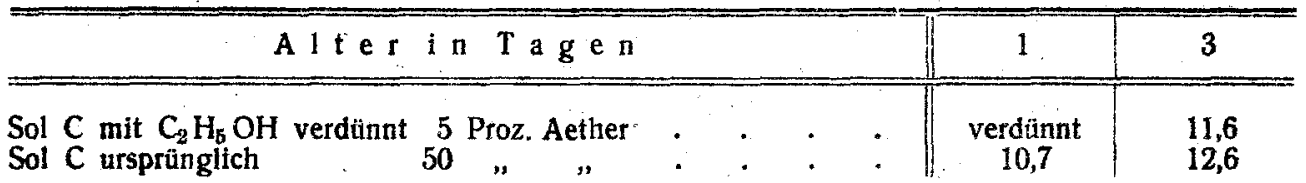

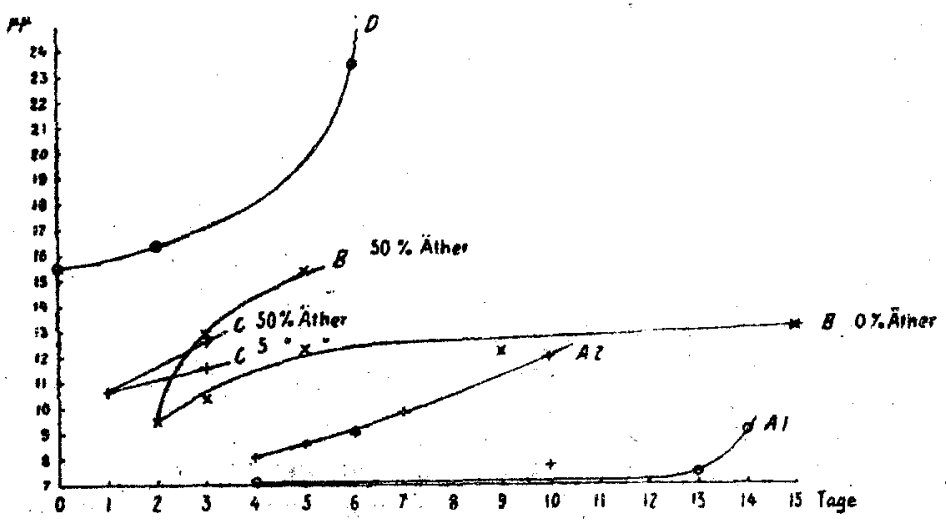

Fig. 1

daß er für falsch angenommen werden mus, aus welchem Grunde, ist unbekannt. Es ist ferner nicht untersucht worden, weshalb $r$ des ursprünglichen Sols $\mathrm{A}$ so abweichend von dem $\mathrm{r}$ der verdünnten Sole $A$ wächst. Im übrigen folgen die Punkte der bestimmten Werte dem Gesetze, daB die TeilchengröBe mit der Zeit - obgleich unregelmäbig und far die verschiedenen Sole verschiedenartig - wachst, und dab eine Vergrößerung des Aethergehalts dieses Wachsen beschleunigt.
Aus der Figur ergibt sich weiter, dah die Kurven der verschiedenen Sole um so böher liegen, je grőfer der Aethergehalt während der Zerstäubung war. (In dieser Beziehung können nur die Sole $A, C$ und $D$ verglichen werden, nur diese sind unter genau denselben Bedingungen hergestellt worden.) Dies kann darauf hindeuten, dab $\mathrm{r}$ - gleich nach der Zerstäubung gemessen - für den größeren Aethergehalt größer ist. Die Versuche der Tabeile V' zeigen die Richtigkeit dieser Annahme.

Die koagulierende Wirkung des Aethers scheint sich also auch wäbrend der Zeit der Zerstäubung geltend zu machen, obwohl hier auferdem übrige Faktoren einspielen können, was jedoch nicht untersucht ist.

Die bis jetzt vorgeführten Versuche sind ganz orientierender Art. Ein wichtiger Faktor für die spontane Koagulation wie die Platinkonzentration ist nicht beachtet worden. Die vorgenommenen Bestimmungen geben in keiner 
Tabelle VI.

Sol E. In einer Mischung von 77 Proz. Aether und 23 Proz. $\mathrm{C}_{2} \mathrm{H}_{5} \mathrm{OH}$ zerstäubt. $\mathrm{Pt}$-Konz. 29,0 mg pro $100 \mathrm{ccm}$. Sedimentationsiemperatur $15^{\circ}$.

Unmitteibar nach Beendigung der Zerstäubung wurde $0,5 \mathrm{ccm}(\mathrm{p}=145,30-3 \mathrm{mg}$ Pt $)$ bei einer Temperatur I $\mathrm{C}$ herausgenommen, $2 \mathrm{ccm} \mathrm{C}_{2} \mathrm{H}_{5} \mathrm{OH}$ hinzugegeben, $10,2 \mathrm{mg}$ Au herausreduziert, nachher Sedimentation.

Sol F. In 77 Proz. Aether zerstäubt. Pt -Konz. $36.0 \mathrm{mg}$ pro $100 \mathrm{ccm}$. Versuch wie bei Sol E.

Sol G. In 84,6 Proz. Aether zerstäubt. Pt-Konz. $28,0 \mathrm{mg}$ pro $100 \mathrm{ccm}$.

Versuch wie bei Sol $E$, jedoch $1 \mathrm{ccm}\left(p=280.10^{-3} \mathrm{mg} P t\right)$ herausgenommen und $4 \mathrm{ccm} \mathrm{C}_{2} \mathrm{H}_{5} \mathrm{OH}$ hinzu. gegeben.

Sol. H. In 98,4 Proz. Aether zerstäubt. Pt-Konz. 16,5 mg pro $100 \mathrm{ccm}$. Versuch wie bei Sol $\mathrm{G}$.

\begin{tabular}{c|c|c|c|c|c|c|c}
\hline Sol & $\begin{array}{c}\text { In Proz. Aether } \\
\text { Zerstäubt }\end{array}$ & $\begin{array}{c}\tau^{0} \mathrm{C} \\
\text { Temperatur bei } \\
\text { der Zerstaubung }\end{array}$ & $\begin{array}{c}\text { Proz. } \\
\mathrm{C}_{2} \mathrm{H}_{5} \text { OH }\end{array}$ & $\sqrt{100 \eta}$ & $1 \mathrm{~mm}$ & h St. & I $\mu \mu$ \\
\hline $\mathrm{E}$ & 77,0 & 77 & 4,2 & 1,19 & 4 & 19,5 & 11,5 \\
$\mathrm{~F}$ & 77,0 & 80 & 4,2 & 1,19 & 4 & 19,5 & 11,9 \\
$\mathrm{G}$ & 84,6 & 80 & 8,4 & 1,30 & 5 & 19 & 16,7 \\
$\mathrm{H}$ & 98,4 & 80 & 8,0 & 1,29 & 17 & 22,5 & 23,7
\end{tabular}

Hinsicht Präzisionswerte. Wegen einer Ainzahl größerer Schwierigkeiten - u. a. mit dem angewendeten Wasser - brachten mehrere angefangene Versuche kein Resultat. Die Zahl der Observationen war deshalb viel zu gering, um alle Zufälligkeiten, die bei derartigen Versuchen unvermeidlich sind, eliminieren zu können. Vor allem sind auberdem die Zahlenergebnisse darum unsicher, weil es selten gelang, Versuche mit verschiedenen Mengen Platin für eine und dieselbe Bestimmung vorzunehmen. Die Resuitate weisen viele Lücken auf und bringen nicht die geplante Beweisführung, welche beabsichtigt war. Aber selbst wenn die einzelneri Werte mit großen Fehlern behaftet sind, zeigen sie doch zusammengenommen eine deutiche Tendenz, die wohl schwer als zufällig angesehen werden kann: Die Sole ändern sich mit der Zeit im Sinne einer Koagulation, und eine Vergröberung des Aether. gehalts beschleunigt diese spontane Koagulation.

\section{Die Temperatur.}

Svedberg ${ }^{13}$ ) erwähnt, dab ein AlkoholAethersol oft koaguliert, wenn die Temperatur erhöht wird. Es ist deshalb zu erwarten, in allen diesbezüglichen Solen einen stets zunehmenden Wert für $r$ zu finden, wenn man, wăhrend die Temperatur steigt, von Zeit zu Zeit Proben herausnimmt. Daraufhin sind einige orientierende Versuche mit Solen bei ca. $\div 80^{\circ}$ dargestellt und mit nachfolgender Erhöhung

13) The Svedberg, loc. cit. der Temperatur vorgenommen worden. Die Tabelle VII ergibt die Resultate.

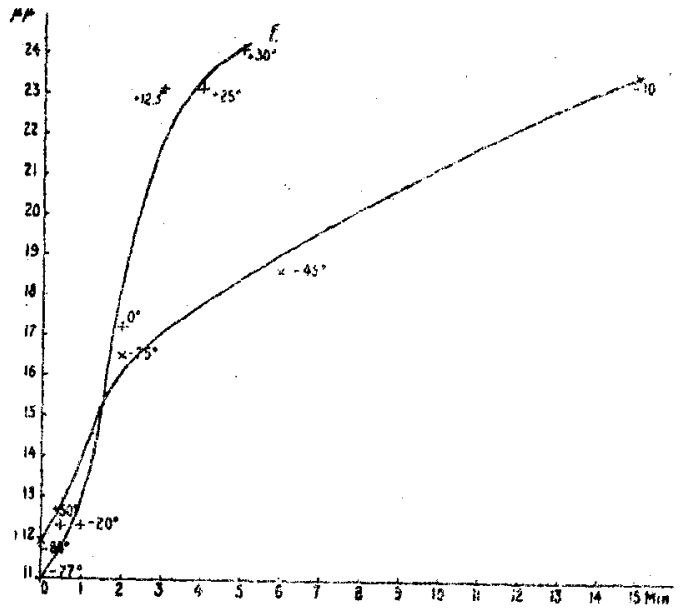

Fig. 2

Die Werte aus Tabeile VII sind in Fig. 2 eingezeichnet, und man sieht, dab unsere Annahme bestätigt ist. Die erhaitenen Zahlenwerte geben allerdings für $r$ bei den angegebenen Temperaturen keinen völlig richtigen Ausdruck. Hierzu sind ganz besondere VorsichtsmaBregeln, die nicht beachtet worden sind, erforderlich. So wird sich z. B. das Sol wahrscheinlich beim Herauspipettieren und beim Ueberführen in den Alkohol, weicher Zimmertemperatur hat, etwas verändern. Die Werte sind auberdem ungenau, weil nur $0,5 \mathrm{ccm}$ herausgenommen ist. Aber an dem Hauptresultat, da $r$ mit $Z$ eit und Temperatur wächst, kann wohl kaum gezweifelt werden. 
Tabelle vil

Sol E. In 77 Proz. Aether zerstäubt. Pt-Konz. $29,0 \mathrm{mg}$ pro $100 \mathrm{~cm}$. Sedimentationstemperatur $15 \%$

Bei den ungefahren Zeiten $t$ Minuten nach Beendigung der Zerstäubung wuràe $0,5 \mathrm{~cm}$ Sol $\mathrm{F}$ i $\mathrm{p}=145.10^{-3} \mathrm{mg} \mathrm{Pt}$ ) herausgenommen, $2 \mathrm{ccm} \mathrm{C}_{2} \mathrm{H}_{5} \mathrm{OH}$ hinzugefugt, $10,2 \mathrm{mg}$ Al herausteduziert, damach Sedimentation in 4,2 Proz. $\mathrm{C}_{2} \mathrm{H}_{5} \mathrm{OH}$, welches bei $15^{i} \sqrt{100} \gamma_{i}=1,19$ entspricht.

Nach der Zerstäubung, weiche bei ca. $-80^{\circ}$ geschahi, wurde die Temperatur dadurch gesteigert, caß das 7erstäubungsgefüß von Wasser mit wechselnder Temperatur ungeben wurde. Die Temperatur $\boldsymbol{x}^{0} \mathrm{C}$ des Sols bei der Zeit $t$ ist in der Tabelle wiedergegeben.

Sol F. In 77 Proz. Aether zerstäubt. Pt-Konz. $36,0 \mathrm{mg}$ pro $100 \mathrm{ccm}$.

Die Versuche wie bei Sol $E$ angegeben, jedoch wurde die Temperatur dadurch erhöht, daß Jas Zerstäubungsgefäß aus der Kältemischung genommen und der Zimmerluft überlassen wurde.

\begin{tabular}{|c|c|c|c|c|c|}
\hline Sol & $\begin{array}{l}\text { Herauspipettiert } \\
\text { bei } a^{0} \mathrm{C}\end{array}$ & $\begin{array}{l}\text { Alter } t \text { Min. beim } \\
\text { Herauspipettieren }\end{array}$ & $1 \mathrm{~mm}$ & h St. & $\mathrm{r} \mu \mu$ \\
\hline $\mathrm{g}$ & $\begin{array}{c}-77 \\
-50 \\
20 \\
0 \\
12,5 \\
25 \\
30\end{array}$ & $\begin{array}{l}0 \\
0,5 \\
1 \\
2 \\
3 \\
4 \\
5\end{array}$ & $\begin{array}{r}4 \\
5 \\
5 \\
10 \\
18 \\
18 \\
19\end{array}$ & $\begin{array}{l}19,5 \\
19,5 \\
19,5 \\
19,5 \\
19,5 \\
19,5 \\
19,5\end{array}$ & $\begin{array}{l}11,0 \\
12,3 \\
12,3 \\
17,2 \\
23,1 \\
23,1 \\
24,1\end{array}$ \\
\hline$F$ & $\begin{array}{r}-80 \\
-75 \\
-45 \\
-10\end{array}$ & $\begin{array}{r}0 \\
2 \\
6 \\
15\end{array}$ & $\begin{array}{r}5 \\
8 \\
10 \\
16\end{array}$ & $\begin{array}{l}19 \\
19 \\
19 \\
19\end{array}$ & $\begin{array}{l}11,9 \\
16,5 \\
18,6 \\
23,5\end{array}$ \\
\hline
\end{tabular}

Bei näherer Betrachtung der beiden Kurven sieht man, daB sie einen ganz verschiedenen Verlauf haben, der nicht allein auf die Ungenauigkeiten der Versuche zurückzuführen ist. Der einzige experimentelle Unterschied zwischen den beiden Versuchsreihen ist, daß die Temperaturerhöhung der Sole abweichend vorgenommen ist, in ersten Falle (Sol $\mathrm{E}$ ) schnell, im andern (Sol F) langsam. Man muf also annehmen, $\mathrm{daB}$ nicht die Temperatur allein für $r$ definierend ist, denn dann würde in beiden Fällen bei derselben Temperatur dasselbe $r$ unabhängig von der Art der Temperaturerhöhung gefunden werden. Bei den Versuchen hingegen ist gefunden :

Für dieselbe Temperatur ergibt sich ein gröberes $r$ bei demjenigen Sol, dessen Alter (vom Anfang der Temperaturerhöhung gerechnet) a $\mathrm{m}$ höchsten ist.

Zurselben $Z$ eit istdas a m grobten bei demjenigen Sol, dessen Temperatur am höchsten ist.

Also: AuBer der Temperatur ist a uch dieZeit - dasAlterdes Kolloids - fürden inneren Zusand eines Sois von Bedtutung.

[Anscheinend sind diese beiden ersten Regeln nicht übereinstimmend mit dem ersten Teil der Kurven, wo die Kurve $F$ oberhalb der Kurve $\mathrm{E}$ liegt Man muf aber in Betracht ziehen, dab der Anfangswert von $r$ in den beiden Fallen verschieden ist; dieses ist dadurch begründet, daB die Konzentrationsbestimmungen für die zwei Sole ein verschiedenes Resultat ergeben haben. Die Konzentrationsbestimmung ist indessen mit grofier Lingenauigkeit behaftet (siehe Abschnitt 4); voraussichtlich ist deshalb die Reproduktion eines Zerstäubens unter genau denselben Bedingungen mit weniger Unsicherheit verbunden. Darum ist es in einem Fall wie diesem besser anzunehmen, daB die Konzentration beider Sole praktisch dieselbe ist, und damit auch die Teilchengröße beim Anfang eines Versuches. Die Form der Kurven wird dadurch nicht verändert, aber die Anfangspunkte werden $\mathbf{z u}$ sammenfallen, und das abengenannte wird besser einleuchten.]

Diese rein orientièrenden Messungen von $r$ zeigen also in die Richtung, dab diese Sole langsamer oder schneller zur Koagulation wandera, je nachdem die Wanderung bei niedriger oder höherer Temperatur geschieht. Also: Man kann auch hier von einer spontanen Koagulation reden. derenGe. schwindigkeit jedoch abnehmen mut, wenn die Temperatur niedrigerwird,

Wir haben hier Teilchengröfemessungen benutzt, um die Wirkung der koagulierenden 
Faktoren zu untersuchen. Man kann natïrlich auch für denselben $Z$ weck die kritische Temperatur messen. Ist ebenerwähntes richtig, so dürfte man erwarten, dab die kritische Temperatur nicht konstant ist, sondern davon abhängt, auf welche Weise die Erhöhung der Temperatur stattfindet, $d . h$. unter welchen Temperaturen der Weg zur vollständigen Koagulation geht.

4. Beobachtungen der "kritischen* Temperatur.

Um diese Sache zu erörtern, sind eine Reihe Bestimmungen der kritischen Temperatur an Solen, welche in kurzer Zeit leicht zur Koagulation gebracht werden können, vorgenommen worden. Die Versuche sind folgendermaBen ausgeführt.

Zerståubung. Die Sole sind alle nach Svedberg's ${ }^{14}$ ) elektrischer Kolloidsynthese mit oszillatorischen Entladungen dargestellt worden. Die Anordnung ist im Wesentlichen dieselbe wie von $S$ vedberg ${ }^{15}$ ) früher beschrieben. Alle bestimmenden Daten sind bei allen Zerstäubungen möglichst unverändert gehalten worden, Die Kapazität des Entladungskreises war $3,2.10^{-3}$ Mikrofarad, Selbstinduktion und Ohm'schen Widerstand praktisch $=0$, die effektive Stromstärke $=1,0$ Ampère, Funkenlănge $=$ ca. $0,08 \mathrm{~mm}$, Volum der Flüssigkeit $60 \mathrm{ccm}$. Die einzigen veränderten $\mathrm{Größen} \mathrm{waren}$ Aethergehalt (der Alkohol-Aether-Mischung) und Zerstäubungsztit. Das Zerstäubungsgefäß war während der Zerstäubung stets von einem Mantel mit einer Kältemischung von fester Kohlensăure und Aether umgeben, wodurch die Zerstäubưng bei ungefähr $-80^{\circ}$ vor sich gehen konnte. Um zu verhindern, dab der Wasserdampf aus der Luft in das Zerstäubungsgefăb eindringen konnte, wurde es vor, während und nach der Zerstäubung möglichst gut mit Watte gedichtet.

Konzentrationsbestimmung. Unmittelbar nachdem die Zerstäubung beendet war, wurden $25 \mathrm{ccm}$ für die Konzentrationsbestimmung herauspipettiert. und in einen gewogenen Tiegel gegeben. Dieser wird zur Verflüchtigung der Flüssigkeit im Luftbad erhitzt. Darauf wird das Residuum sehr vorsichtig geglüht, denri die bei der Zerstäubung gebildeten Teerstoffe verursachen während der Destruktion kleine Exsplosionen. Der Rückstand von Platin betrug $2-7 \mathrm{mg}$. 1908).

14) The Svedberg, loc. cit.

15) The Svedberg, Koil. - Zeitschr. 2, 142 (1907)
Bestimmung der Koagulationste m peratur. 9 Minuten nach Beendigung des Zerstäubens wurde das Zerstäubungsgefäb aus der Kältemischung herausgenommen und in ein Bad von Alkohol oder Wasser hineingetaucht. Die Temperatur des Bades beim Eintauchen wurde in den verschiedenen Versuchen variiert (z. B. für die niedrigen Temperaturen mittelst Alkohol durch feste Kohlensäure). Da das Flüssigkeitsvolum in Bade gegen 2 Liter betrug, war die Temperaturemiedrigung während eines Versuches sehr klein, gewöhnlich nur ein Paar Grade. In einigen Fällen wurde als" Bad nur Luft von Zimmertemperatur angewendet. Das Zerstäubungsgefä $B$ wurde im Bade in einer schrägen Stellung angebracht, aber so flach wie möglich. Unter demselben befand sich eine kleine elektrische Lampe, damit Veränderungen im Sol leicht zu sehen waren. Es wurde stets langsam mit einem Toluolthermometer umgerührt. In dem Augenblick der Koagulation wurde das Thermometer abgelesen, und die Zeit vom Eintauchen an gerechnet mit Hilfe einer gewöhnlichen Stoppuhr observiert.

Wenn die Temperatur durch Alkohol- oder Wasserbad erhöht wird, findet die sichtbare Koagulation rein momentan statt. Dies ist nicht bei der langsamen Temperatursteigerung im Luftbade der Fall; hier kann deutlich eine allmähliche Koagulation von kleineren zu größeren Komplexen wahrgenommen werden. Eingenauer Koagulationspunkt kann deshalb bei dieser makroskopischen Methode nicht angegeben werden, die Beobachtungen gelten deswegen der ersten Andeutung zur Koagulation. Die Resultate finden sich in Tabelle VIII zusammengestellt,

Bei Nr. 1-23 ist käuflicher Aether pro narcosi angewendet. Dieser ist voratissichtlich schwach wasserhaltig, und daher konnen die Resultate nicht mit Nr. 24-34 verglichen werden, wo der Aether absolut wasserfrei ist, über Natrium destilliert.

Bei Nr. 1, 4 und $6-12$ ist die Platinkonzenfration nicht direkt bestimmt worden, weil man voraussetzen kann, daß Zerstäubungen mit gleichem Erfolge reproduziert werden körnen. Hiervon bekommt man einen Eindruck, wenn man die folgende Tabelle IX betrachtet, diese enthält ein Verzeichnis über sämtliche Zerstäubungen, bei denen eine Konzentrationsbestimmung vorgenommen worden ist.

Wenn man sich dessen erinnert, dab nur 2-7 mg Platin eingewogen wurden und übrige Versuchsfehler gerade bei dieser Bestimmung 
Tabelle VIII.

A. Versuche, wo Aether pro narcosi verwendet wurde.

\begin{tabular}{|c|c|c|c|c|c|c|c|c|c|c|c|}
\hline Sol & $\begin{array}{l}\text { In Proz. } \\
\text { Aether } \\
\text { zerstäubt }\end{array}$ & $\begin{array}{c}\text { Pt-Kanz. } \\
\text { mig pro } \\
100 \text { cenz } \\
\text { gewogen }\end{array}$ & $\begin{array}{c}\mathrm{Pt}-\text { Eonz } \\
\mathrm{mg} \text { pro } \\
100 \mathrm{ccm} \\
\text { berechnet }\end{array}$ & $\begin{array}{c}\begin{array}{c}\text { Zerstäu- } \\
\text { bungs- } \\
\text { zeit } \\
\text { Min. }\end{array} \\
\end{array}$ & $\begin{array}{c}\text { Zerstalu- } \\
\text { bungs- } \\
\text { tempera- } \\
\text { tur }{ }^{\circ} \mathrm{C} \\
\div \\
\end{array}$ & $\begin{array}{c}\text { Temperatur- } \\
\text { steige- } \\
\text { rungs- } \\
\text { bad }\end{array}$ & $\begin{array}{c}\text { Tempe- } \\
\text { ratur des } \\
\text { Bades } \\
0^{\circ} \mathrm{C}\end{array}$ & $\begin{array}{l}\text { Koag.: } \\
\text { Tempe- } \\
\text { ratur } \\
{ }^{\circ} \mathrm{C}\end{array}$ & $\begin{array}{l}\text { Alter bei } \\
\text { der Koag. } \\
\text { pom Anfang } \\
\text { der Tem- } \\
\text { peratur- } \\
\text { erhbhung } \\
\text { Sel. } \\
\end{array}$ & $\begin{array}{l}\text { Durchschn.- } \\
\text { Gesehwin- } \\
\text { digkeit der } \\
\text { Temporatur- } \\
\text { steigerung } \\
\frac{\Delta t}{\Delta t} \cdot \mathrm{C} \\
\end{array}$ & $\begin{array}{l}\text { Durchschn. } \\
\text { Aufonthalts- } \\
\text { dauer im } \\
\text { Temperatur- } \\
\text { Intervall } \\
\frac{\Delta t}{\Delta \tau} \text { Sek. } \\
\end{array}$ \\
\hline \begin{tabular}{l|l}
1 & $a_{1}$ \\
2 & $a_{1}$ \\
3 & $a_{1}$ \\
4 & $a_{1}$ \\
5 & $a_{1}$ \\
6 & $a_{1}$ \\
7 & $a_{1}$ \\
8 & $a_{1}$ \\
9 & $a_{1}$
\end{tabular} & $\begin{array}{l}96,9 \\
96,9 \\
96,9 \\
96,9 \\
96,9 \\
96,9 \\
96,9 \\
96,9 \\
96,9\end{array}$ & $\begin{array}{l}\overline{20,0} \\
18,0 \\
\overline{18,0} \\
= \\
=\end{array}$ & $\begin{array}{l}19,8 \\
19,8 \\
19,8 \\
19,8 \\
19,8 \\
19,8 \\
19,8 \\
19,8 \\
19,8\end{array}$ & $\begin{array}{l}15 \\
15 \\
15 \\
15 \\
15 \\
15 \\
15 \\
15 \\
15\end{array}$ & $\begin{array}{l}80 \\
30 \\
75 \\
80 \\
80 \\
80 \\
80 \\
80 \\
80\end{array}$ & $\begin{array}{c}\text { Wasser } \\
\text { "' } \\
\text { Alkohol } \\
\text { Wasser } \\
\text { Alkohol } \\
\text { Luft }\end{array}$ & $\begin{array}{r}37 \\
33 \\
27 \\
15 \\
0 \\
0 \\
2 \\
-33 \\
16\end{array}$ & $\begin{array}{l}-10 \\
-15 \\
=17 \\
=21 \\
-24 \\
-25 \\
-29 \\
-45 \\
-47\end{array}$ & $\begin{array}{r}67 . \\
76 \\
72 \\
\cdot 90 \\
81 \\
98 \\
95 \\
197 \\
305\end{array}$ & $\begin{array}{l}1,04 \\
0,85 \\
0,80 \\
0,65 \\
0,69 \\
0,62 \\
0,54 \\
0,18 \\
0,11\end{array}$ & $\begin{array}{l}0,96 \\
1,18 \\
1,25 \\
1,54 \\
1,45 \\
1,61 \\
1,85 \\
5,62 \\
9,26\end{array}$ \\
\hline \begin{tabular}{l|l}
10 & $a_{2}$ \\
11 & $a_{g}$ \\
12 & $a_{2}$
\end{tabular} & $\begin{array}{l}96,9 \\
96,9 \\
96,9\end{array}$ & $\frac{-}{-}$ & $\begin{array}{l}13,2 \\
13,2 \\
13,2\end{array}$ & $\begin{array}{l}10 \\
10 \\
10\end{array}$ & $\begin{array}{l}80 \\
79 \\
79\end{array}$ & $\begin{array}{c}\text { Alkohol } \\
\text { Luft }\end{array}$ & $\begin{array}{r}13 \\
-10 \\
16\end{array}$ & $\begin{array}{l}-16 \\
-30 \\
-45\end{array}$ & $\begin{array}{l}120 \\
150 \\
322\end{array}$ & $\begin{array}{l}0,53 \\
0,33 \\
0,11\end{array}$ & $\begin{array}{l}1,89 \\
3,30 \\
9,50\end{array}$ \\
\hline \begin{tabular}{l|l}
13 & $b_{1}$ \\
14 & $b_{1}$ \\
15 & $b_{1}$
\end{tabular} & $\begin{array}{l}92,3 \\
92,3 \\
92,3\end{array}$ & $\begin{array}{l}28,4 \\
28,4 \\
30,0\end{array}$ & $\begin{array}{l}28,9 \\
28,9 \\
28,9\end{array}$ & $\begin{array}{l}15 \\
15 \\
15\end{array}$ & $\begin{array}{l}79 \\
78 \\
79\end{array}$ & $\begin{array}{c}\text { Wasser } \\
\text { Luft }\end{array}$ & $\begin{array}{r}33 \\
0 \\
16\end{array}$ & $\begin{array}{r}+7 \\
-14 \\
-32\end{array}$ & $\begin{array}{r}78 \\
108 \\
518\end{array}$ & $\begin{array}{l}1,10 \\
0,59 \\
0,09\end{array}$ & $\begin{array}{c}0,91 \\
1,69 \\
11,1\end{array}$ \\
\hline \begin{tabular}{l|l|l}
16 & $b_{2}$ \\
17 & $b_{2}$ \\
18 & $b_{2}$
\end{tabular} & $\begin{array}{l}92,3 \\
92,3 \\
92,3\end{array}$ & $\begin{array}{l}20,4 \\
20,8 \\
21,4\end{array}$ & $\begin{array}{l}20,9 \\
20,9 \\
20,9\end{array}$ & $\begin{array}{l}10 \\
10 \\
10\end{array}$ & $\begin{array}{l}78 \\
78 \\
79\end{array}$ & $\begin{array}{c}\text { Wasser } \\
\text { Lüt }\end{array}$ & $\begin{array}{r}36 \\
0 \\
16\end{array}$ & $\begin{array}{r}+21 \\
-6 \\
-32\end{array}$ & $\begin{array}{l}120 \\
185 \\
480\end{array}$ & $\begin{array}{l}0,82 \\
0,39 \\
0,10\end{array}$ & $\begin{array}{c}1,22 \\
2,56 \\
10,0\end{array}$ \\
\hline $19 \mathrm{c}$ & $\begin{array}{l}97,9 \\
97,9 \\
97,9\end{array}$ & $\begin{array}{l}13,6 \\
17,2 \\
21,6\end{array}$ & $\begin{array}{l}17,5 \\
17,5 \\
17,5\end{array}$ & $\begin{array}{l}15 \\
15 \\
15\end{array}$ & $\begin{array}{l}78 \\
78 \\
77\end{array}$ & $\begin{array}{c}\text { Wasser } \\
\text { Luft }\end{array}$ & $\begin{array}{r}34 \\
0 \\
16\end{array}$ & $\begin{array}{l}-23 \\
-35 \\
-73\end{array}$ & $\begin{array}{l}43 \\
50 \\
60\end{array}$ & $\begin{array}{l}1,28 \\
0,86 \\
0,07\end{array}$ & $\begin{array}{c}0,78 \\
1,16 \\
14,9\end{array}$ \\
\hline $\begin{array}{l}22 \\
23\end{array}$ & $\begin{array}{l}84,6 \\
84,6\end{array}$ & $\begin{array}{l}33,0 \\
34,0\end{array}$ & $\begin{array}{l}31,4 \\
31,4\end{array}$ & $\begin{array}{l}15 \\
15\end{array}$ & $\begin{array}{l}80 \\
80\end{array}$ & $\begin{array}{c}\text { Wasser } \\
\text { Luft }\end{array}$ & $\begin{array}{l}16 \\
16\end{array}$ & $\begin{array}{l}+1 \\
-25\end{array}$ & $\begin{array}{l}181 \\
645\end{array}$ & $\begin{array}{l}0,45 \\
0,09\end{array}$ & $\begin{array}{c}2,22 \\
11,7\end{array}$ \\
\hline
\end{tabular}

B. Versuche, wo Aether über Natrium destilliert verwendet wurde.

\begin{tabular}{|c|c|c|c|c|c|c|c|c|c|c|c|}
\hline \begin{tabular}{l|l}
24 & $e_{1}$ \\
25 & $e_{1}$ \\
26 & $e_{1}$ \\
27 & $e_{1}$
\end{tabular} & $\begin{array}{l}92,3 \\
92,3 \\
92,3 \\
92,3\end{array}$ & $\begin{array}{c}24,4 \\
22,8 \\
24,4\end{array}$ & $\begin{array}{l}23,9 \\
23,9 \\
23,9 \\
23,9\end{array}$ & $\begin{array}{l}15 \\
15 \\
15 \\
15\end{array}$ & $\begin{array}{l}76 \\
78 \\
79 \\
76\end{array}$ & $\begin{array}{l}\text { Wasser } \\
\text { Alkohol } \\
\text { Luft }\end{array}$ & $\begin{array}{r}0 \\
15 \\
-11 \\
16\end{array}$ & $\begin{array}{l}-26 \\
-26 \\
-35 \\
-58\end{array}$ & $\begin{array}{r}72 \\
75 \\
140 \\
180\end{array}$ & $\begin{array}{l}0,69 \\
0,69 \\
0,31 \\
0: 10\end{array}$ & $\begin{array}{r}1,45 \\
1,45 \\
3,22 \\
10,0\end{array}$ \\
\hline & $\begin{array}{l}92,3 \\
92,3 \\
92,3\end{array}$ & $\begin{array}{l}16,8 \\
16,4 \\
17,2\end{array}$ & $\begin{array}{l}16,8 \\
16,8 \\
16,8\end{array}$ & $\begin{array}{l}10 \\
10 \\
10\end{array}$ & $\begin{array}{l}77 \\
77 \\
78\end{array}$ & $\begin{array}{c}\text { Wasser } \\
\text { Alkohol } \\
\text { Luft }\end{array}$ & $\begin{array}{r}15 \\
-8 \\
16\end{array}$ & $\begin{array}{l}-14 \\
-27 \\
-49\end{array}$ & $\begin{array}{r}95 \\
175 \\
275\end{array}$ & $\begin{array}{l}0,66 \\
0,29 \\
0,11\end{array}$ & $\begin{array}{l}1,52 \\
3,45 \\
9,5\end{array}$ \\
\hline $\begin{array}{l}e_{5} \\
e_{3} \\
e_{9}\end{array} \mid$ & $\begin{array}{l}92,3 \\
92,3 \\
92,3\end{array}$ & $\begin{array}{l}13,2 \\
14,4 \\
14,8\end{array}$ & $\begin{array}{l}14,1 \\
14,1 \\
14,1\end{array}$ & $\begin{array}{l}7,5 \\
7,5 \\
7,5\end{array}$ & $\begin{array}{l}77 \\
76 \\
76\end{array}$ & $\begin{array}{c}\text { Wasser } \\
\text { Alkohol } \\
\text { Luft }\end{array}$ & $\begin{array}{r}15 \\
0 \\
16\end{array}$ & $\begin{array}{r}+2 \\
\pm 10 \\
-28\end{array}$ & $\begin{array}{l}175 \\
272 \\
480\end{array}$ & $\begin{array}{l}0,45 \\
0,24 \\
0,10\end{array}$ & $\begin{array}{r}2,22 \\
4,16 \\
10,0\end{array}$ \\
\hline 1 & $\begin{array}{l}95,4 \\
84,6\end{array}$ & $\begin{array}{l}23,2 \\
30,0\end{array}$ & 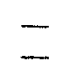 & $\begin{array}{l}15 \\
15\end{array}$ & $\begin{array}{l}78 \\
78\end{array}$ & Wasser & $\begin{array}{l}36 \\
38\end{array}$ & $\begin{array}{r}-35 \\
+15\end{array}$ & $\begin{array}{r}38 \\
130\end{array}$ & $\begin{array}{l}1,13 \\
0,71\end{array}$ & $\begin{array}{l}0,89 \\
1,41\end{array}$ \\
\hline
\end{tabular}

*) Unter der Rubrik: „Pt-Konz. berechnet" sind die Durchschnittswerte, welche aus Tabelle IX ersichtlicn sind, angegeben.

ziemlich grof sind, wird man verstehen, dab es gestattet sein mul - wie es im Folgenden getan wird - Sole zu vergleichen, welche in gleicher Zeit und im übrigen unter genau denselben Bedingungen zerstäubt sind, obschon die Konzentrationsbestimmungen ein etwas abweichendes Resultat ergaben. Die Platinkonzentration bei $\mathrm{Nr} .1,4,6,7,8$ und 9 ist hiernach auf $19,8 \mathrm{mg}$ zu setzen, gleich dem Durchschnittswerte aller Zerstäubungen, in dem betreffenden Aethergehalt wăhrend 15 Minuten vorgenommen.

In Tabelle IX befinden sich außerdem Bestimmungen von zerstäubter Masse während vers chi edener Zeiten, aber sonst unter denselben Bedingungen erhalten. Die Angaben gelten besonders Solen mit 92,3 Proz. Aether. (Tab. IX B resp. VIII B), diese betreffend sind die Resultate aus Figur 3 ersichtlich. 
Tabelle IX.

A. Versuche, wo Aether pro narcosi serwerdet wurde.

\begin{tabular}{|c|c|c|c|c|c|}
\hline $\begin{array}{l}S_{\text {Sol }} \\
\text {. }\end{array}$ & $\begin{array}{l}\text { In Froz: } \\
\text { Aether } \\
\text { zex- } \\
\text { stäubt }\end{array}$ & $\begin{array}{l}\text { Zersi.- } \\
\text { Zeit } \\
\text { Min. }\end{array}$ & $\begin{array}{c}\text { Pi - Konz. } \\
\text { mp pro } \\
100 \text { cem } \\
\text { gawogenz }\end{array}$ & 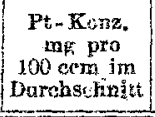 & Anmerkuag \\
\hline 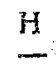 & $\begin{array}{l}98,4 \\
98,4\end{array}$ & $\begin{array}{l}15 \\
15\end{array}$ & $\begin{array}{l}16,5 \\
15,5\end{array}$ & 16,0 & \\
\hline $\begin{array}{l}19 \\
20 \\
21\end{array}$ & $\begin{array}{l}97,9 \\
97,9 \\
97,9\end{array}$ & $\begin{array}{l}15 \\
15 \\
15\end{array}$ & $\begin{array}{l}13,6 \\
17,2 \\
21,6\end{array}$ & 17,5 & $\begin{array}{l}\text { Die Eletieroden } \\
\text { batter sieh } \\
\text { eivas yerschoben }\end{array}$ \\
\hline $\begin{array}{r}2 \\
3 \\
5 \\
- \\
-\end{array}$ & $\begin{array}{l}96,9 \\
96,9 \\
96,9 \\
96,9 \\
96,9\end{array}$ & $\begin{array}{l}15 \\
15 \\
15 \\
15 \\
15\end{array}$ & $\begin{array}{l}20,0 \\
18,0 \\
18,0 \\
21,0 \\
22,0\end{array}$ & 19,8 & \\
\hline $\begin{array}{l}13 \\
14 \\
15\end{array}$ & $\begin{array}{l}92,3 \\
92,3 \\
92,3\end{array}$ & $\begin{array}{l}15 \\
15 \\
15\end{array}$ & $\begin{array}{l}28,4 \\
28,4 \\
30,0\end{array}$ & 28,9 & \\
\hline $\begin{array}{l}16 \\
17 \\
18\end{array}$ & $\begin{array}{l}92,3 \\
92,3 \\
92,3\end{array}$ & $\begin{array}{l}10 \\
10 \\
10\end{array}$ & $\begin{array}{l}20,4 \\
20,8 \\
21,4\end{array}$ & 21,4 & \\
\hline $\begin{array}{l}22 \\
23 \\
G \\
\end{array}$ & $\begin{array}{l}86,4 \\
86,4 \\
86,4 \\
86,4\end{array}$ & $\begin{array}{l}15 \\
15 \\
15 \\
15\end{array}$ & $\begin{array}{l}33,0 \\
34,0 \\
28,0 \\
30,5\end{array}$ & 31,4 & \\
\hline$\frac{D}{\mathrm{E}}$ & $\begin{array}{l}77,0 \\
77,0 \\
77,0\end{array}$ & $\begin{array}{l}15 \\
15 \\
15\end{array}$ & $\begin{array}{l}32,0 \\
36,0 \\
29,0\end{array}$ & 32,3 & \\
\hline$c$ & 50,0 & 15 & 24,5 & -- & \\
\hline A & 0,0 & 15 & 15,5 & $=$ & \\
\hline
\end{tabular}

B. Versuche, wo Aether über Natrium destilliert verwendet wurde.

Sol Nr. $24-33$ siehe Tabelle VIIIB, die särntliche Zablenwerte enthalt.

\section{\begin{tabular}{lll|l|l}
$-\mid 92,3$ & 5 & 10,2 & $\ldots$
\end{tabular}}

Mit dem Zeichen - ist angegeben, då das betreffende Sol in dieser Arbeit nicht verwendet wotden ist.

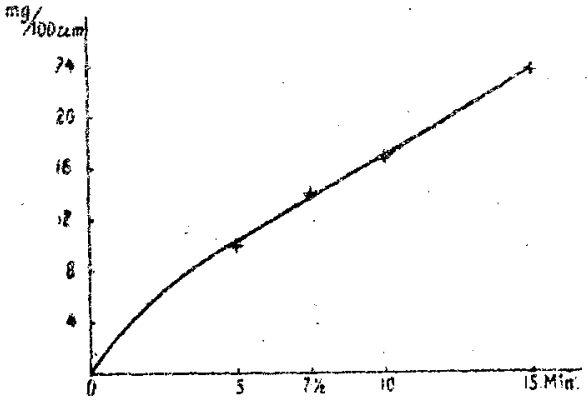

Fig. 3

Die Variation mit der Zeit ist also für diesen über Natrium destillierten Aether annähernd linear, wenn die Zeit nicht gar $z u$ kurz ist. Man mu daher die Konzentration der Zerstäubunger Nr. 10, 11 und 12 in Aether pro narcosi (während 10 Minuten) gieich zweidrittel des obgenannten Durchschnitswertes $=\frac{2}{3} \cdot 19,8 \mathrm{mg}=13,2 \mathrm{mg}$ setzen hönnen. (Die Reiation $3: 2$ jat doch nicht ganz bei Nr. 13-15 im Vergleich zu Nr. $16-18$ erfülit).

In Nr. 19, 20, 21 waren die Platinelektroden etwas ungleichmäBig abgenutzt, der Wert dieser Versuche ist daher möglicherojeise nicht so sehr grob.

Besprechung der Resultate Die Tabelle VIIl zeigt unmittelbar, dab die kritische Temperatur für mitelnander $z u$ vergleichende Sole (d.h. Sole, die unter scharf denselben Bedingungen dargestellr sind), nicht konstant ist, wie man früher annahm. Die. Ursache dieses Ergebnisses muB in dern einzigen variierten Faktor bei den Versuchen gesehen werden, nämlich dem Charakier und der Tem. peratur des Erwärmungsbades, also der Geschwindigkeit, mit welcher die Temperatur des Sols zum Steigen gebracht wird. Diese Geschwindigkeit $\frac{\mathrm{d} \Theta}{\mathrm{dt}}$ (wo $\Theta$ die absolute Tempesatur und $t$ die Zeit ist), ist natürlich nicht ganz konstant während der Zeit, wo die Temperatur bis zum Koagulationspunkte steigt, sondern varilert gemâb $\mathrm{N}$ ew to n's Abkuhhungsgesetz. Hier ist indessen eine besimmte Zah! wünschenswert, welche die Temperatursteigerung jeder einzelnen beobachteten kritischen Temperatur charakterisieven kann. Man kann - urn eine Uebersicht zu bekommen - den Durchschnittswert der yerschiedenen Erwärmungsgeschwindigkeiten in Laure der Versuchszeit $t$ annähernd benutzen. Der Durchschnittswert wird

$$
\frac{1}{t} \int_{0}^{t}\left(\frac{d \theta}{d t}\right) \cdot d t=\frac{1}{t} \int_{0}^{t} d \theta=\frac{1}{t}\left(\theta_{k}-\theta\right)
$$

wo $\Theta_{k}$ die kritische Temperatur und $\Theta_{0}$ die Eintauchtemperatur im Bade ist (absolut gerechnet). Die gesuchte Größe ist also die durchschnittliche Temperatursteigerung in der Zeiteinheit, in der Tabelle VIll unter $\frac{\Delta \tau}{\Delta t}$ wiedergegeben. ( $x$ ist Temperatur in Graden $C$.) Wird diese als Abszisse benutzt, und ist die dazu gehörige kritische Temperatur als Ordínate, so ergibt sich Pig. 4 u. 5. 


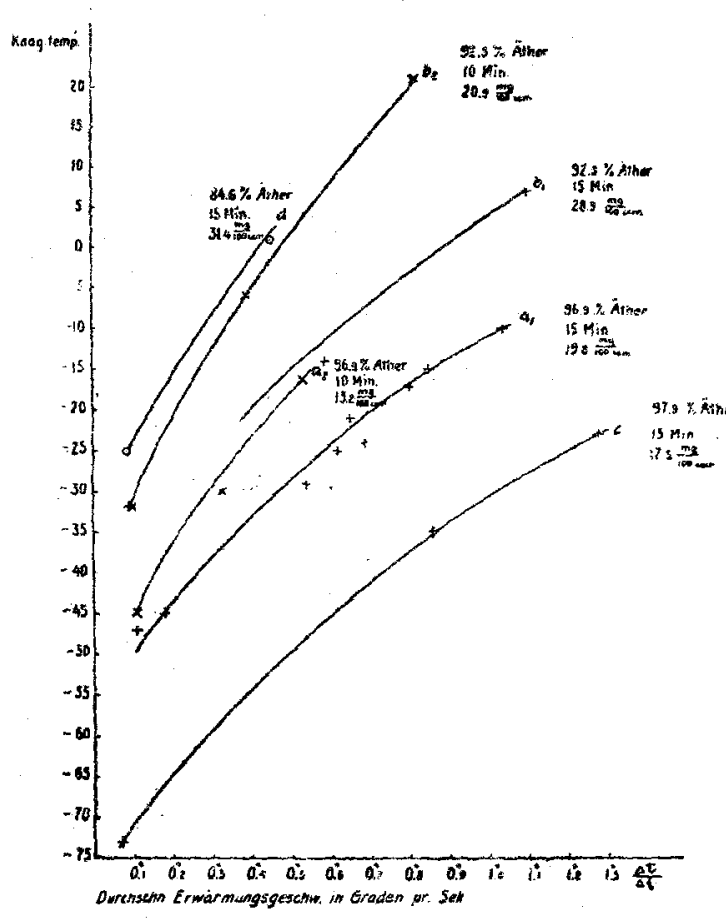

Fig. 4

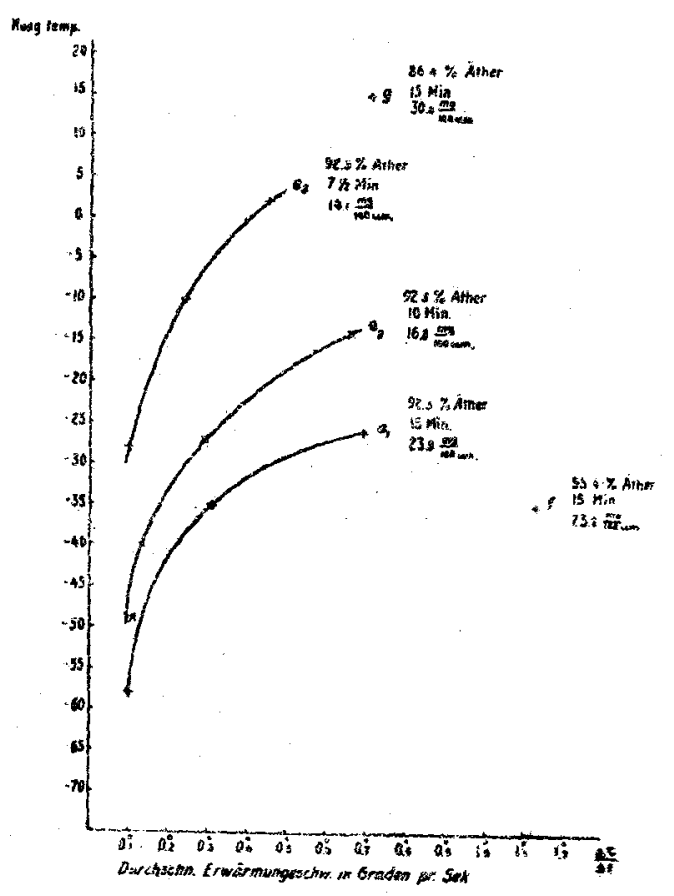

Fig. 5.

In diesen Figuren tritt deutlich folgende GesetzmäBigkeit hervor:

Je gröber die Erwärmungsgeschwindigkeit, desto höher die kritische Temperatur.
Bei Betrachtung der Kurven darf man jedoch nicht vergessen, daB die angewendeten Abszissen durchaus keine homogene Charakteristik der verschiedenen Erwärmungen geben, denn liegt die kritische Temperatur derjenigen des Bades besonders nahe, so ist die Abszisse zu klein, ist die Koagulationstemperatur besonders weit von derjenigen des Bades entfernt, ist sie zu groh. Die Abweichung der Punkte von einer kontinuierlichen Kurve beruht deshalb nicht allein auf Versuchsfehlern. Diese können allerdings recht bedeutend sein, worüber die verschiedenen Versuche Nr. $1-9$ AufschluB geben können.

Da die Zeitdauer, um die spontane Koagulation zu Ende zu bringen, in den verschiedenen Versuchen so ganz verschieden ist, wird man verstehen, dab das. Umrühren kein wesentlicher koagulierender Faktor ist. Wäre dieses der Fall, könnte man eine vollstândige Koagulation nach ziemlich denselben Zeiten, von der Erwärmungsart unabhängig, erwarten.

Der reziproke Wert der durchschnittlichen Erwärmungsgeschwindigkeit wird $\frac{\Delta t}{\Delta \tau}$ oder die durchschnittliche Zeit, welche das Sol gebraucht um um $1^{0}$ erwärmt $z u$ werden. Die Gröbe kann als ein annäherndes $\mathrm{MaB}$ angesehen werden für die durchschnittliche Zeitdauer der Aufenthalte des Sols bei jedem Temperaturintervall, während es gegen Koagulation wandert. Die Beziehung zwischen Koagulationstemperatur und "Aufenthaltszeit" ist für einige der beobachteten Serien in Figur 6 und 7 dargestellt.

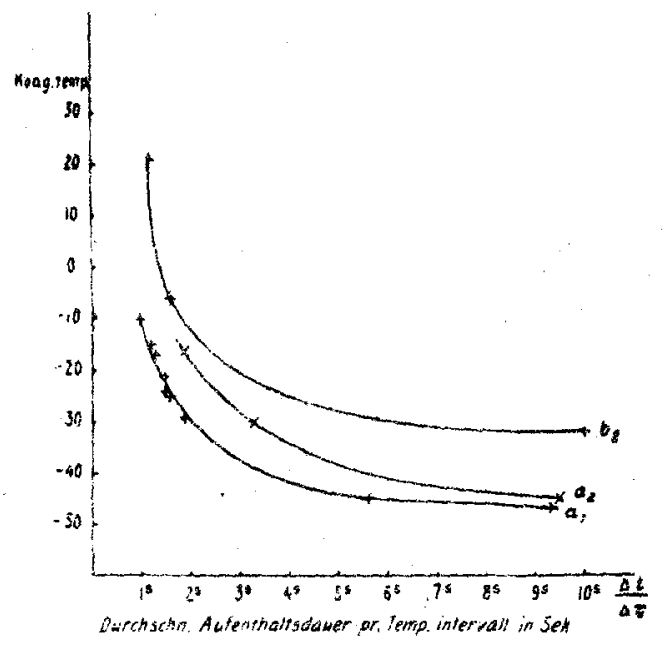

Fig. 6

Hieraus geht die letzterwähnte Gesetzmäßigkeit in einer anderen Weise hervor: 


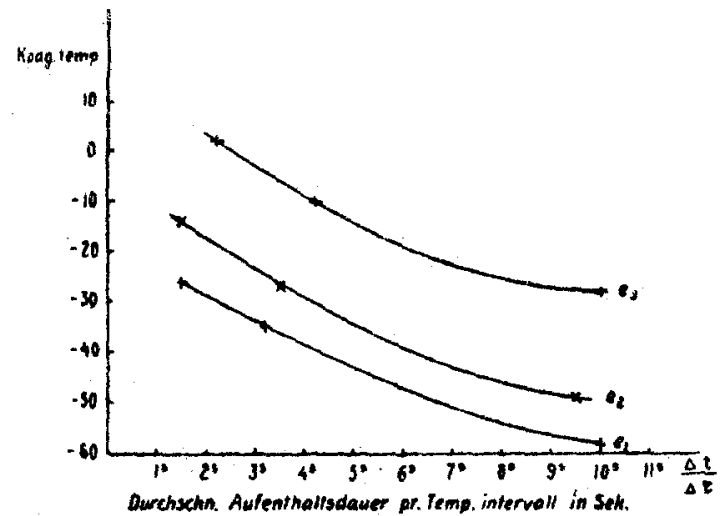

Fig. 7

Je niedriger die kritische Temperatur, desto längeredurchschnittliche Aufenthaltszeit bei jedem Temperaturintervall.

Letzteres zwingt zur Annahme, daB die niedrigeren Temperaturen pro Zeiteinheit weniger $z$ ur Koagulation -beitragen" als die höheren.

Von dem Standpunkt der oben dargebotenen Auffassung aus betrachtet, dab die diesbezüglichen Sole, selbst bei konstanter Temperatur, stets gegen Koagulation wandern - also die spontane Koagulation - wird man darauf gebracht, das die Geschwindigkeit der spontanen Koagulation von der Temperatur abhägt, indem niedrigere Temperaturen einegeringere Geschwindigkeit bedingen.

Der wenig scharf definierte Koagulationspunkt bei der langsamen Erwärmung im Luftbade ist gerade eine sichtbare Uebereinstimmung mit dieser Gesetzmäßigkeit.

Die Zeit von der Darstellung eines Sols bis zur Koagulation wird natũrlich die Le be n sdauer des Kolloids genannt werden können. Aus obenstehendem könnte man daher schließen, daß die Lebensdauer eines Sols um so länger ist, unter je niedrigerer Temperatur es „gelebt" hat.

Es ist interessant, dieses Resultat mit der Koagulationstheorie von $\mathrm{S} \mathrm{mol} \mathrm{u} \mathrm{ch} \mathrm{ow} \mathrm{s} \mathrm{k} \mathrm{i}{ }^{16}$ ) $\mathrm{zu}$ vergleichen, obschon dieselbe nicht für diesbezügliche Koagulationen ausgearbeitet ist. Nacb dieser Theorie ist die Koagulationszeit (hier die Zeit, bis die ursprüngliche Anzahl Teilchen $\nu_{0}$ bis zur Hälfte verringert wird) bei konstanter Temperatur gleich:

16) M. v. Smoluchow ski, loc. cit.

$$
\frac{1}{4 \pi \mathrm{D} \varrho \nu_{0}}
$$

wo $\varrho$ der Radius der Attraktionssphäre ist und $D$ der Diffusionskoeffizient für die B rown'sche Bewegung. Nach den Untersuchungen von A. Einstein ${ }^{17}$ ) ist

wo

$$
\mathrm{D}=\frac{\mathrm{R} \Theta}{\mathrm{N}} \cdot \frac{1}{6 \pi \eta \mathrm{r}}
$$

$R$ die Gaskonstante,

$\mathrm{N}$ die wirkliche Anzahl der Moleküle im Grammolekül,

$r$ der Radius der Teilchen,

$\theta$ die absolute Temperatur und

$\eta$ die Viskosität des Dispersionsmittels.

Nun variiert $\eta$ sehr stark mit der Temperatur, aber man kennt keine exakte GesetzmäBigkeit für dessen Abnahme bei steigender Temperatur. Man kann indessen setzen

$$
\eta=\frac{k}{\theta^{n}}
$$

wo $k$ eine Konstante ist, und n eine (wahrscheinlich variable) Zahl gröBer als 1 [ $\mathrm{Bat}$ s $\mathrm{ch}$ in s k i ${ }^{18}$ ) gibt $n=3$ an]. Wenn man von der Variation des $r$ und $\rho$ absieht, kann man also sagen, daß die Koagulationszeit $\Theta^{n+1}$ umgekehrt proportional ist, ein Resultat, welches in qualitativer Uebereinstimmung mit den Konsequenzen der vorgenommenen Versuche ist. Hiermit ist nichts von den Faktoren gesagt, welche bei einer Theorie für gerade solche Koagulationen in Betracht kommen.

Eine vollzogene Koagulation, durch steigende Temperaturen hindurch, kann nun als $\mathrm{Zu}$ sammenfassung aller kleinen "Beiträge" zur Koagulation aufgefaft werden, welche während des "Aufenthalts" des Sols bei den verschiedenen Temperaturintervallen geleistet werden, indem diese "Beiträge " pro Zeiteinheit weniger "effektiv* sind, je niedriger die Temperatur ist.

Die Tatsache, daB ein Kolloid, welches durch Temperatursteigerung zur Koagulation gebracht werden kann, eine Koagulationstemperatur hat, die von der Art der Temperaturerhöhung abhängig ist, ist eine nicht unbekannte Erscheinung in der Kolloidchemie. Chick und Martin ${ }^{19}$ ) haben diesbezüglich gezeigt, dab die Koagulationstemperatur der Eiweißstoffe nicht - wie allgemein angenommen - eine

17) A. Einstein, Drude's Ann. d. Physik 17, 549 (1905).

18) Batschinski, Beibl. 25, 231, 789 (1901).

19) Chick und Martin. Journ. of Physiol. 40, 404 (1910). 
für den berreffenden Eiweißstoff charakteristische Konstante ist, sondern mit der Temperatur des Erwärmungsbades variiert: Weiter zeigen sie, $\mathrm{da} B$ die Koagulation mit um so geringerer Geschwindigkeit vor sich geht, je niedriger die Temperatur des Erhitzungsbades ist. Also haben wilr hier ganz analoge Verhältnisse wie bei den Platinkollaiden (obwohl die Eiweißkoagulation nicht allein auf ein Zusammentreten von Teilchen zurückzuführen ist ${ }^{20}$ ).

Bei Vergleichung solcher, ,kritischen “ Kurven in Figur 4 und 5, wo die Aetherkonzentration konstant ist, während die Platinkonzentration variiert (also $a_{1}$ und $a_{2}, b_{1}$ und $b_{2}, e_{1}, e_{2}$ und $e_{3}$ ), sieht man, dab diejenigen Kurven, welche der größten Platinkonzentration entsprechen, am niedrigsten liegen ( $a_{1}$ liegt niedriger als $a_{2}, b_{1}$ niedriger als $b_{2}, e_{1}$ niedriger als $e_{2}, e_{2}$ niedriger als $e_{3}$ ). Man kann also sagen :

Bei konstanter Ae therkonzentration (im übrigen unter denselben Bedingungen) wird die spontane Koagulation schneller vor sich gehen, wenn die platinkonzentration vergröbert wird.

Diese GesetzmäBigkeit ist eine Erweiterung einer bereits von $S$ vedber ${ }^{20}$ ) angedeuteten, dab die kritische Temperatur bei steigender Platinkonzentration herabgedrückt wird. Da er bei allen Versuchen mit einer ziemlich gleich großen Erwärmungsgeschwindigkeit gearbeitet hat, entsprechen seine Resultate denjenigen, welche aus den Durchschnittspunkten zwischen den kritischen Kurven in Fig. 4 und 5 und einer Ordinate mit einer seiner Erwärmungsgeschwindigkeit entsprechenden Abszisse abgeleitet werden können.

Wenn man eine gleiche Zeit lang, aber in Plüssigkeiten von verschiedener Aetherkonzen ${ }^{*}$ tration, zerstäubt, ergibt sich, wie Tabelle IX zeigt, daß die Masse des zerstäubten Platins mit steigendem Aethergehalt abnimmt (die größeren Aetherkonzentrationen betreffend). Die Resultate für die Zerstäubung während 15 Minuten sind in Figur 8 wiedergegeben.

Vergleicht man die kritischen Kurvan zweier Sole mit verschiedenem Aethergehalt (aber

20) Svedberg hat schon in Svensk Kemisk Tidskrift Nr. 6 (1908), erwähnt, daß zwischen einigen Platinorganosolen und EiweiBstoffen insofern eine Analogie besteht, als beide eine kritische Temperatur erweisen. Aus Obenstehendem geht hervor, daß diese Análogie nun bedeutend weiter zu führen ist.

23) loc. cit. während gleicher Zeit zerstäubt), so könnte man erwarten, daß dasjenige Sol mit dem größeren Aethergehalt der höherliegenden Kurve entspricht, weil die Platinkonzentration hier am geringsten' ist. Nichtsdestoweniger bedingt ein größerer Aethergehalt eine niedriger liegende Kurve. Mit andern Worten:

Eine Vergrößerung der Aetherkonzentrationbeschleunigt die Geschwindigkeit derautomatischen Koagulation, eine Tatsache, die schon früher angedeutet worden ist (Abschnitt 2).

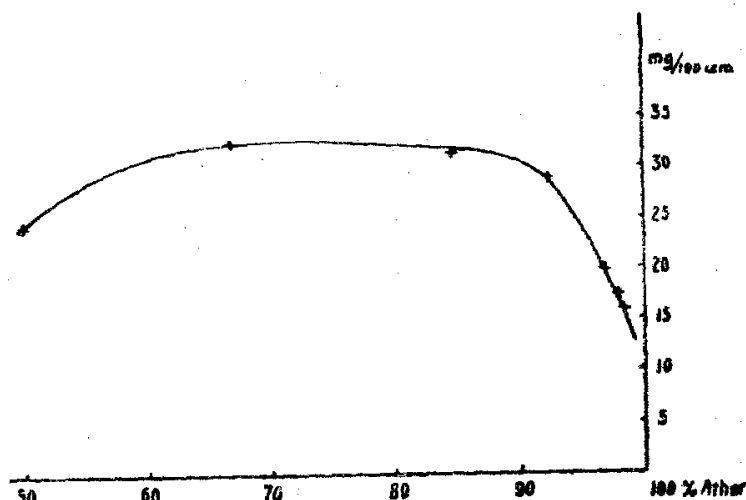

Fig. 8

Zusammenfassung.

1. Es ist nachgewiesen worden, daB Platinamikronen vergoldet werden können, sowohl wenn das Dispersionsmittel absoluter Alkohol ist, als auch Mischungen von Aether und Alkohol. Es ist ferner nachgewiesen worden, dab der durchschnittliche Radius der Amikronen dann gefunden werden kann, wenn der Radius der vergoldeten Teilchen durch Sedimentation bestimmt wird. Ist Aether zugegen, muß jedoch die Sedimentation in ganz geschlossenen Gefäben vor sich gehen, da sonst die Verdampfung des Aethers die Sedimentation verhindert.

2. Die Stabilitätsverhältnisse bei Platinsolen mit Aether-Alkoholmischungen als Dispersionsmittel sind untersucht worden, sowohl mit Hilfe von Teilchengröbebestimmnngen, wie auch durch Beobachtungen von Koagulationstemperaturen.

3. Es zeigt sich, daß man annehmen mub, dat diese Sole nie ganz stabil sind, sondern stets - anscheinend spontan - zur vollständigen Koagulation wandern. Das Alter des Sols ist also für seine Charakteristik maligebend.

4. Die Temperatur, die Platinkonzentration und die Aetherkonzentration scheinen, von einander unabhängig, die $\mathrm{G}$ e s chwindigk e it dieser Wanderung zu beeinflussen, und zwar 
so, daß die Geschwindigkeit zunimmt, wenn die Gröbe eines oder mehrerer dieser Faktoren zunimmt.

5. Der Begriff "Die kritische Temperatur“ eines solchen Sols kann in seiner ursprünglichen Gestalt nicht länger aufrecht erhalten werden, denn es gibt keine konstante obere Temperaturgrenze für die Existernz des Sols. Die Koagulationstemperatur hăngt vielmehr von der Geschwinäigkeit $a b$, mit welcher die Temperatur des Sols zum Steigen gebracht wird. Es ist deshalb besser, von einer fur das betrefiende Sol "kritischen Kurve" zu sprechen.

Die Untersuchungen werden fortgesetzt.

Upsala und Kristiania 1920

Chemisches Universitätslaboratorium.

\section{Chemische Reaktionen in Solgemischen.}

\section{Von H. Freundlich und Alexander Nathansohn.}

(Eingegangen am 9. März 1921.)

Die in der vorangehenden Mitteilung ${ }^{1}$ ) beschriebene Wechselwirkung zwischen zwei negativen Solen, dem $\mathrm{As}_{2} \mathrm{~S}_{3}$ - und dem S-Sol, regte dazu an, Versuche mit anderen Gemischen negativ geladener Sole anzustellen. Es zeigten sich dabei Erscheinungen, die - obgleich noch aicht mit voller Sicherheit zu deuten -- doch schon jetzt einer vorläufigen Mitteilung wert sinả.

Dazu gehört vor allem die Reaktion zwischen enem in der oben angegebeinen Weise hergesteliten Odén'schen Schwefelsol und einem nach Carey Lea - durch Reduktion mit Ferrosulfat und Natriumzitrat - bereiteten Silbersol. Mischt man z. B. $5 \mathrm{ccm}$ eines Schwefelsols von ca. $500 \mathrm{mg}$ S.Gehalt im Liter mit $1 \mathrm{ccm}$ graugrünlichen Silbersols von $400 \mathrm{mg}$ Ag im Liter, so färbt sich beim Vermischen die Flüssigkeit braun und macht dann eine ganze Kette von Farbenänderungen durch; nach etwa 1 Minute ist sie weinrot, nach $1 \frac{1 / 2}{2}$ Minuten geht sie ins violette über, nach $2^{1 / 2}$ Minuten ist sie trefviolett und wird dabei sehr klar, nach 4 Minuten fängt sie an zu verblassen, nach 5 Minuten ist sie stahiblau, nach 8 Minuten grüpblau, nach 10 Minuten nimnt sie die grünbrautse Farbe verwelkenden Laubes an, von da an verblabt sie weiter. Nach 20 Minuten ist noch ein grünicher Farbton zu sehen, nach ca. 25 Minuten hat sie eine blabgelbbraune Färbung angenommen, die sich nicht weiter verändert. Koaguliert man während dieser Farbänderungen $\mathrm{zu}$ irgendeinem Zeitpunkte das Sol mit Aluminiumsalz, so haben die entstehenden Flocken die jeweilige Farbe des Sols und machen dann dieselbe Kette von Farbänderungen durch wie jenes, nur meist etwas langsamer, so dab nach etwa einer Stunde die Endfarbe erreicht ist.

1) Koll.-Zeitschr. 28, 258 (1921).
Es liegt natürlich die Annahme nahe, dab im Schwefelsol etwas $\mathrm{H}_{2} \mathrm{~S}$ enthalten ist, und dak es sich hier um eine Reaktion des kolloiden Silbers mit dem Schwefelwasserstoff landelt; und dies um so mehr, als man tatsächlich beim Vermischen des Silbersols mit Schwefelwasserstoffwasser verschiedener Konzentration (das gesättigte $\mathrm{H}_{2} \mathrm{~S}$. Wasser wurde auf $1 / 10$ bis $1 / 100$ verdünnt) Farbveränderungen ähnlicher Art findet, deren Auftreten, Wechsel und Aufeinanderfolge im ganzen aber weniger regelmäbig ist als bei den erwähnten Solgemischen. Die Erscheinungen, die man bei Anwendung von Schwefelwassersioffwasser sieht, stellen ein verblaßites Abbild derer dar, die man an dem Solgemisch beobachtet.

Aber diese Annahme ist nicht ohne weiteres statthaft. Wie in der vorangehenden Abhandlung erwahnt wurde, reagiert $\mathrm{H}_{2} \mathrm{~S}$ mit der Pentathionsäure des Schwefelsols. ist die Konzentration des $\mathrm{H}_{2} \mathrm{~S}$ hoch genug, so wird das ursprünglich gelblich durchsichtige Sol weik und undurchsichtig und läbt einen nicht wieder peptisierbaren Schwefel fallen. Bei kleineren $\mathrm{H}_{2} \mathrm{~S}$-Konzentrationen bleibt es äuberlich unverändert und zum Sol zugesetztes $\mathrm{H}_{2} \mathrm{~S}$ läßt sich auch nach längerer Zeit an der Rotfärbung mit Nitroprussidnatrium nachweisen. Wie weit es zum Teil noch mit den Mizellen reagiert, etwa in lockere Verbindungen übergegangen ist, wurde noch nicht geprüft. Jedenfalls hat man ein Recht, nur sehr Wleine Konzentrationen von $\mathrm{H}_{2} \mathrm{~S}$ im Schwefelsol anzunehmen.

Nun spricht ein Umstand dafür, daß es sich gar nicht um eine Reaktion zwischen Schwefelwasserstoff und Silbermizellen handelt, sondern tatsächlich um eine zwischen Mizellen des Schwefels und denen des Silbers. Dies wäre natürlich recht beachtenswert, weil es sich ja 